\title{
A criterion for good reduction of Drinfeld modules and Anderson motives in terms of local shtukas
}

\author{
URS HARTL AND SIMON HÜSKEN
}

\begin{abstract}
For an Anderson $A$-motive over a discretely valued field whose residue field has $A$-characteristic $\varepsilon$, we prove a criterion for good reduction in terms of its associated local shtuka at $\varepsilon$. This yields a criterion for good reduction of Drinfeld modules. Our criterion is the function-field analog of Grothendieck's [15, Proposition IX.5.13] and de Jong's [19, 2.5] criterion for good reduction of an Abelian variety over a discretely valued field with residue characteristic $p$ in terms of its associated $p$-divisible group
\end{abstract}

Mathematics Subject Classification (2010): 11G09 (primary); 145L05 (secondary).

\section{Introduction}

We fix a finite field $\mathbb{F}$ with $r$ elements and characteristic $p$. Let $\mathcal{C}$ be a smooth projective and geometrically irreducible curve over $\mathbb{F}$ with function field $Q=\mathbb{F}(\mathcal{C})$. Let $\infty \in \mathcal{C}$ be a closed point and let $A=\Gamma\left(\mathcal{C} \backslash\{\infty\}, \mathcal{O}_{\mathcal{C}}\right)$ be the $\mathbb{F}$-algebra of those rational functions on $\mathcal{C}$ which are regular outside $\infty$. For every $\mathbb{F}$-algebra $R$ we let $\sigma$ be the endomorphism of $A_{R}:=A \otimes_{\mathbb{F}} R$ given by $\sigma:=\operatorname{id}_{A} \otimes \operatorname{Frob}_{r, R}: a \otimes b \mapsto$ $a \otimes b^{r}$ for $a \in A$ and $b \in R$.

Let $o_{L}$ be a complete discrete valuation ring containing $\mathbb{F}$, with fraction field $L$, uniformizing parameter $\pi$, maximal ideal $\mathfrak{m}_{L}=(\pi)$ and residue field $\ell=o_{L} / \mathfrak{m}_{L}$. We assume that $\ell$ is a finite field extension of $\ell^{p}$. This is equivalent to saying that $\ell$ has a finite $p$-basis over $\ell^{p}$ in the sense of [7, Section V.13, Definition 1]. It holds for example if $\ell$ is perfect, or if $\ell$ is a finitely generated field. Since every Anderson $A$-motive over $L$ can be defined over a finitely generated subfield of $L$ our restriction on $\ell$ is not serious. Let $c^{*}: A \rightarrow o_{L}$ be a homomorphism of $\mathbb{F}$-algebras such that the kernel of the composition $A \rightarrow o_{L} \rightarrow \ell$ is a maximal

We thank the Deutsche Forschungsgemeinschaft for supporting this research in form of SFB 878. Received April 25, 2013; accepted in revised form January 16, 2014.

Published online February 2016. 
ideal $\varepsilon$ in $A$. We say that the residue field $\ell$ has finite $A$-characteristic $\varepsilon$. We do not assume that $c^{*}: A \rightarrow o_{L}$ is injective. So $L$ can have either generic $A$ characteristic $\operatorname{ker} c^{*}=(0)$ or finite $A$-characteristic $\operatorname{ker} c^{*}=\varepsilon$. In the following we will consider various $A_{o_{L}}$-algebras. In all of them we consider the ideal generated by $\left\{a \otimes 1-1 \otimes c^{*}(a): a \in A\right\} \subset A_{o_{L}}$. By abuse of notation we denote all these ideals by $\mathfrak{J}$.

By an Anderson A-motive over $L$ we mean a pair $\underline{M}=\left(M, F_{M}\right)$ consisting of a locally free $A_{L}$-module $M$ of finite rank, and an injective $A_{L}$-homomorphism $F_{M}: \sigma^{*} M \rightarrow M$ where $\sigma^{*} M:=M \otimes_{A_{L}, \sigma} A_{L}$, such that $\operatorname{coker}\left(F_{M}\right)$ is a finite dimensional $L$-vector space and is annihilated by a power of $\mathfrak{J}$. We say that $\underline{M}$ has good reduction over $o_{L}$ if there exists a locally free $A_{o_{L}}$-module $\mathcal{M}$ and an injective $A_{o_{L}}$-homomorphism $F_{\mathcal{M}}: \sigma^{*} \mathcal{M} \rightarrow \mathcal{M}$ such that $\left(\mathcal{M}, F_{\mathcal{M}}\right) \otimes_{A_{o_{L}}} A_{L} \cong \underline{M}$ and $\operatorname{coker}\left(F_{\mathcal{M}}\right)$ is a finite free $o_{L}$-module which is annihilated by a power of $\mathfrak{J}$. We call $\underline{M}=\left(\mathcal{M}, F_{\mathcal{M}}\right)$ a good model of $\underline{M}$. In particular if $\underline{M}=\underline{M}(\phi)$ is the Anderson $A$ motive associated with a Drinfeld $A$-module $\phi$ over $L$, then $\underline{M}$ has good reduction if and only if $\phi$ has good reduction; see Proposition 4.10.

Anderson $A$-motives are function-field analogs of Abelian varieties. For an Abelian variety $\mathcal{A}$ over a discretely valued field $K$ with residue field of characteristic $p$ there are criteria for good reduction in terms of local data. For a prime number $l \neq p$ the criterion of Néron-Ogg-Shavarevich [22, Section 1, Theorem 1] states that $\mathcal{A}$ has good reduction if and only if the $l$-adic Tate module $T_{l} \mathcal{A}$ of $\mathcal{A}$ is unramified as a $\operatorname{Gal}\left(K^{\mathrm{alg}} / K\right)$-representation. At the prime $p$ the criterion of Grothendieck [15, Proposition IX.5.13] (for $\operatorname{char}(K)=0$ ), respectively de Jong $[19,2.5]$ (for $\operatorname{char}(K)=p$ ) states that $\mathcal{A}$ has good reduction if and only if the Barsotti-Tate group $\mathcal{A}\left[p^{\infty}\right]$ has good reduction.

These criteria have function-field analogs for Anderson $A$-motives. The ana$\log$ of the Néron-Ogg-Shavarevich-criterion was proved by Gardeyn [12, Theorem 1.1]. In this article we simultaneously prove the analog of Grothendieck's and de Jong's criterion. Here the function-field analogs of Barsotti-Tate groups are local shtukas [17, Section 2.1] which are defined as follows. Let $A_{o_{L},(\varepsilon, \pi)}$ be the $(\varepsilon, \pi)$-adic completion of $A_{o_{L}}$. An (effective) local shtuka at $\varepsilon$ over $o_{L}$ is a pair

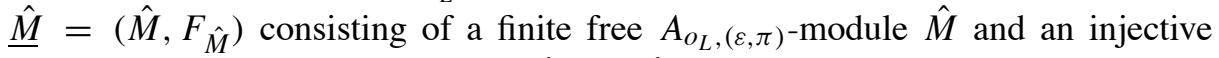
$A_{o_{L},(\varepsilon, \pi)}$-homomorphism $F_{\hat{M}}: \sigma^{*} \hat{M} \rightarrow \hat{M}$ such that $\operatorname{coker}\left(F_{\hat{M}}\right)$ is a finite free $o_{L}$-module and is annihilated by a power of $\mathfrak{J}$. The local shtuka associated with a good model $\underline{\mathcal{M}}$ of an Anderson $A$-motive is $\underline{\hat{M}}(\underline{\mathcal{M}}):=\underline{\mathcal{M}} \otimes_{A_{o_{L}}} A_{o_{L},(\varepsilon, \pi)}$. Strictly speaking effective local shtukas are the function field analogs of the $F$-crystals of Barsotti-Tate groups. The analogs of the latter are called $\varepsilon$-divisible local Andersonmodules and their category is equivalent to the category of effective local shtukas; see [18] for more details. Our analog of Grothendieck's and de Jong's reduction criterion is now the following:

Corollary 6.6. Let $\underline{M}$ be an Anderson A-motive over L. Then $\underline{M}$ has good reduction over $o_{L}$ if and only if there is an effective local shtuka $\underline{\hat{M}}$ at $\varepsilon$ over $o_{L}$ and an isomorphism $\underline{M} \otimes_{A_{L}} A_{o_{L},(\varepsilon, \pi)}[1 / \pi] \cong \underline{\hat{M}} \otimes_{A_{o_{L},(\varepsilon, \pi)}} A_{o_{L},(\varepsilon, \pi)}[1 / \pi]$. 
(In the body of the text we prove a slightly stronger statement.) This applies in particular if $\underline{M}$ is the Anderson $A$-motive associated with a Drinfeld module $\phi$ over $L$ to give a criterion for good reduction of $\phi$ in terms of its associated local shtuka. The reformulation of this criterion in terms of the $\varepsilon$-divisible local Anderson-module of $\phi$ is given in [18].

ACKNOWLEDGEMENTS. We would like to thank the anonymous referee for his careful reading and for asking an interesting question which lead to the answer given in Remark 5.4.

\section{The base rings}

Let $o_{L}$ be an equi-characteristic complete discrete valuation ring containing the finite field $\mathbb{F}$, with quotient field $L=\operatorname{Frac}\left(o_{L}\right)$ and residue field $\ell=o_{L} / \mathfrak{m}_{L}$, where $\mathfrak{m}_{L} \subset o_{L}$ is the maximal ideal of $o_{L}$. We assume that $\ell$ is a finite field extension of $\ell^{p}:=\left\{b^{p}: b \in \ell\right\}$. We fix a uniformizer $\pi=\pi_{L}$ of $o_{L}$ and sometimes identify $o_{L}$ with $\ell \llbracket \pi \rrbracket$. Let $v=v_{\pi}=\operatorname{ord}_{\pi}(\cdot)$ be the discrete valuation on $L$ normalized by $v(\pi)=1$.

We assume that there is an $o_{L}$-valued point $c \in \mathcal{C}\left(o_{L}\right)$ such that the corresponding $\mathbb{F}$-morphism $c: \operatorname{Spec}\left(o_{L}\right) \rightarrow \mathcal{C}$ factors via $\mathcal{C} \backslash\{\infty\} \subset \mathcal{C}$. Such a datum corresponds to a homomorphism of $\mathbb{F}$-algebras $c^{*}: A \rightarrow o_{L}$ which we call the characteristic map. We further assume that the closed point $V(\pi) \subset \operatorname{Spec}\left(o_{L}\right)$ is mapped to a closed point $\varepsilon$ of $\operatorname{Spec}(A) \subset \mathcal{C}$. The latter is the kernel of the composition $A \rightarrow o_{L} \rightarrow \ell$. So, in accordance with Drinfeld's terminology [9], we call $\varepsilon$ the residue characteristic or residual characteristic place of $Q$. By continuity, the characteristic map $c^{*}: A \rightarrow o_{L}$ factors through a morphism of complete discrete valuation rings $A_{\varepsilon} \rightarrow o_{L}$ where $A_{\varepsilon}$ is the completion of $A$ at the characteristic place $\varepsilon$. Note that $A_{\varepsilon} \rightarrow o_{L}$ is injective if $c^{*}$ is injective, and factors through $A / \varepsilon$ if $c^{*}$ is not injective.

Remark 2.1. Since $A$ is a Dedekind domain there is a power $\varepsilon^{m}$ which is a principal ideal in $A$. We fix a generator $t$ of $\varepsilon^{m}$ and frequently use the finite flat monomorphism of $\mathbb{F}$-algebras $\iota: \mathbb{F}[z] \rightarrow A, z \mapsto t$.

For any $\mathbb{F}$-algebra $R$ we abbreviate $A_{R}:=A \otimes_{\mathbb{F}} R$. In particular, $A_{o_{L}} \subset A_{L}$ is a noetherian integral domain, and by virtue of the equality $A_{\ell} \cong A_{o_{L}} / \pi A_{o_{L}}$ it follows that $\pi \in o_{L}$ is a prime element of $A_{o_{L}}$.

Definition 2.2. Let $A_{o_{L}, \pi}$ (respectively, $A_{o_{L},(\varepsilon, \pi)}$ ) be the completion of the $o_{L^{-}}$ algebra $A_{o_{L}}$ for the $\pi$-adic topology (respectively, the $(\varepsilon, \pi)$-adic topology).

By Krull's Theorem ([8], III.3.2), the ring $A_{o_{L}}$ is separated for both the $\pi$-adic and the $(\varepsilon, \pi)$-adic topology. The topological $o_{L}$-algebra $A_{o_{L}, \pi}$ is admissible in the sense of Raynaud, i.e. it is of topologically finite presentation and has no $\pi$-torsion. In particular, the $L$-algebra $A_{o_{L}, \pi}[1 / \pi]$ is affinoid in the sense of rigid analytic geometry; see [4-6]. 
For example if $\mathcal{C}=\mathbb{P}_{\mathbb{F}}^{1}$ and $A=\mathbb{F}[z]$ then we have $A_{o_{L}}=o_{L}[z]$ and correspondingly $A_{L}=L[z]$. Let us specify that $\varepsilon=z \mathbb{F}[z]$. Our choice of a uniformizer $\pi$ gives rise to an identification $o_{L}=\ell \llbracket \pi \rrbracket$. Consequently $o_{L} \llbracket z \rrbracket=\ell \llbracket \pi \rrbracket \llbracket z \rrbracket=$ $\ell \llbracket \pi, z \rrbracket=A_{o_{L},(\varepsilon, \pi)}$. On the other hand, the $\pi$-adic completion of $o_{L}[z]$ equals $o_{L}\langle z\rangle:=\left\{\sum_{i=0}^{\infty} b_{i} z^{i}: v\left(b_{i}\right) \rightarrow \infty(i \rightarrow \infty)\right\}$, and since $L\langle z\rangle=o_{L}\langle z\rangle \otimes_{o_{L}} L$, we may view $A_{O_{L}, \pi}[1 / \pi]$ as a replacement, for general $\mathcal{C}$, of the Tate algebra $L\langle z\rangle$ of strictly convergent power series in one indeterminate $z$ over $L$, which serves as coordinate ring for the one-dimensional affinoid unit ball in rigid analytic geometry.

There is a natural embedding $A_{L} \rightarrow A_{o_{L}, \pi}[1 / \pi]$ which, for general $\mathcal{C}$, replaces the completion homomorphism $L[z] \rightarrow L\langle z\rangle$, and which itself can be regarded as a completion map with respect to the $L$-algebra norm-topology on the reduced affinoid $L$-algebra $A_{o_{L}, \pi}[1 / \pi]$ and its restriction on $A_{L}$; see [4, Section 1.4, Proposition 19]. Note that the canonical homomorphism $A_{o_{L}} \rightarrow A_{o_{L},(\varepsilon, \pi)}$ factors uniquely via $A_{o_{L}, \pi}$, where the induced map $A_{o_{L}, \pi} \rightarrow A_{o_{L},(\varepsilon, \pi)}$ identifies $A_{o_{L},(\varepsilon, \pi)}$ with the $(\varepsilon, \pi) A_{o_{L}, \pi}$-adic completion of $A_{o_{L}, \pi}$. Since $A_{o_{L}, \pi}$ is a regular integral domain, it is $(\varepsilon, \pi) A_{o_{L}, \pi}$-adically separated by Krull's theorem and $A_{o_{L}, \pi} \rightarrow A_{o_{L},(\varepsilon, \pi)}$ is injective and flat.

Recall that there is a finite flat monomorphism of $\mathbb{F}$-algebras $\iota: \mathbb{F}[z] \rightarrow A$ which identifies the indeterminate $z$ with the generator $t \in A$ of $\varepsilon^{m}$ chosen in Remark 2.1. The $o_{L}$-algebra homomorphism $\iota \otimes \mathrm{id}: o_{L}[z] \rightarrow A_{o_{L}}, \sum_{\nu} a_{\nu} z^{\nu} \mapsto$ $\sum_{v} t^{\nu} \otimes a_{v}$, is finite flat, so that we obtain finite flat maps

$$
o_{L}\langle z\rangle \rightarrow A_{o_{L}, \pi}, \quad L\langle z\rangle \rightarrow A_{o_{L}, \pi}[1 / \pi], \quad o_{L} \llbracket z \rrbracket \rightarrow A_{o_{L},(t, \pi)}, \quad \ell[z] \rightarrow A_{\ell}
$$

Here the $(t, \pi)$-adic completion $A_{o_{L},(t, \pi)}$ of $A_{o_{L}}$ equals $A_{o_{L},(\varepsilon, \pi)}$ since $(\varepsilon, \pi)^{m} \subset$ $\left(\varepsilon^{m}, \pi\right)=(t, \pi)$ in $A_{o_{L}}$.

Lemma 2.3. If $A_{o_{L}, \varepsilon}$ denotes the $\varepsilon$-adic completion of $A_{o_{L}}$, the canonical map $A_{o_{L}, \varepsilon} \rightarrow A_{o_{L},(\varepsilon, \pi)}$ is an isomorphism.

\section{Frobenius modules}

The $r$-Frobenius Frob Fro $_{L} \rightarrow o_{L}, x \mapsto x^{r}$, gives rise to an endomorphism

$$
\sigma=\mathrm{id}_{A} \otimes \operatorname{Frob}_{r}: A_{o_{L}} \rightarrow A_{o_{L}}, \quad a \otimes x \mapsto a \otimes x^{r}
$$

which extends to give a map $\mathrm{id}_{A} \otimes \mathrm{Frob}_{r, L}: A_{L} \rightarrow A_{L}$ again denoted by $\sigma$. On the other hand, reducing mod $\pi$ gives $\bar{\sigma}=\mathrm{id}_{A} \otimes \mathrm{Frob}_{r, \ell}: A_{\ell} \rightarrow A_{\ell}$. The latter is a finite flat endomorphism of the Dedekind domain $A_{\ell}$, because $\ell$ is finite over $\ell^{p}$. The map $\sigma: A_{o_{L}} \rightarrow A_{o_{L}}$ is $\pi$-adically and $(\varepsilon, \pi)$-adically continuous and therefore extends to give endomorphisms $A_{o_{L}, \pi} \rightarrow A_{o_{L}, \pi}$ and $A_{o_{L},(\varepsilon, \pi)} \rightarrow A_{o_{L},(\varepsilon, \pi)}$, again denoted by $\sigma$. 
Lemma 3.1. In the commutative diagram

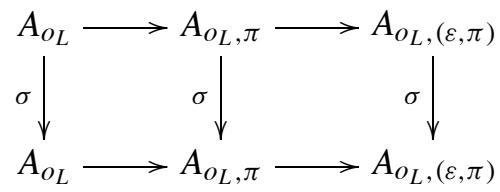

both squares are co-Cartesian, and the vertical arrows are finite flat.

We let the proof be preceded by the following:

Remark 3.2. Via the identification $o_{L}=\ell \llbracket \pi \rrbracket$, the $r$-Frobenius Frob ${ }_{r, o_{L}}: o_{L} \rightarrow$ $o_{L}$ is mirrored by the map $\ell \llbracket \pi \rrbracket \rightarrow \ell \llbracket \pi \rrbracket, \sum_{\nu=0}^{\infty} a_{\nu} \pi^{v} \mapsto \sum_{\nu=0}^{\infty} a_{v}^{r} \pi^{r \nu}$. Choosing an $\ell^{r}$-basis of $\ell$ and lifting it to a subset $W$ of $o_{L}$, this implies $\left(\operatorname{Frob}_{r, o_{L}}\right)_{*} o_{L}=$ $\bigoplus_{i=0}^{r-1} \bigoplus_{w \in W} o_{L} w \pi^{i}$, so that $\operatorname{Frob}_{r, o_{L}}: o_{L} \rightarrow o_{L}$ is finite flat.

Proof of Lemma 3.1. By base change the remark implies that $\sigma=\mathrm{id}_{A} \otimes$ Frob $_{r, o_{L}}$ : $A_{o_{L}} \rightarrow A_{o_{L}}$ is finite flat, and that $A_{o_{L}} \otimes_{\sigma, A_{o_{L}}} A_{o_{L}, \pi}$ is a finite flat $A_{o_{L}, \pi}$-module and hence equals the $\pi$-adic completion of the $A_{o_{L}}$-module $\sigma_{*} A_{o_{L}}$. If we let $\mathfrak{a}=$ $\sigma\left(\pi A_{o_{L}}\right) A_{o_{L}}=\pi^{r} A_{o_{L}}$ and $\mathfrak{b}=\pi A_{o_{L}}$, we get $\mathfrak{b}^{r}=\mathfrak{a} \subset \mathfrak{b}$. Consequently, by [10, Lemma 7.14], the inverse systems $\left(A_{o_{L}} / \mathfrak{a}^{n}\right)_{n}$ and $\left(A_{o_{L}} / \mathfrak{b}^{n}\right)_{n}$ give the same limit, which shows that the square on the left is co-Cartesian, and that $\sigma: A_{o_{L}, \pi} \rightarrow$ $A_{o_{L}, \pi}$ is finite flat. Similarly, we have $\sigma(\varepsilon, \pi) A_{o_{L}}=\left(\varepsilon, \pi^{r}\right) \subset(\varepsilon, \pi)$ as well as $(\varepsilon, \pi)^{r} \subset\left(\varepsilon, \pi^{r}\right)$, which proves that the displayed diagram qualifies $A_{o_{L},(\varepsilon, \pi)}$ as tensor product $A_{o_{L},(\varepsilon, \pi)} \otimes_{A_{o_{L}}, \sigma} A_{o_{L}}$, and that $\sigma: A_{o_{L},(\varepsilon, \pi)} \rightarrow A_{o_{L},(\varepsilon, \pi)}$ is finite flat.

Finally, note that the embedding of $o_{L}$-algebras $\iota \otimes$ id $: o_{L}[z] \rightarrow A_{o_{L}}$ commutes with $\sigma: A_{o_{L}} \rightarrow A_{o_{L}}$ and the $r$-Frobenius lift of $o_{L}[z]$, given by $o_{L}[z] \rightarrow$ $o_{L}[z], \quad \sum_{v} a_{v} z^{\nu} \mapsto \sum_{\nu} a_{v}^{r} z^{\nu}$. Consequently, also the embeddings from (2.1) are Frobenius-equivariant.

Let $B$ be an $o_{L}$-algebra together with a ring endomorphism $\sigma: B \rightarrow B$ such that $\sigma$ and $\mathrm{Frob}_{r, o_{L}}: o_{L} \rightarrow o_{L}$ are compatible with the structure map $o_{L} \rightarrow B$. For example, $B$ could be any of the base rings considered above.

Definition 3.3. We define the category $\mathrm{FMod}(B)$ of Frobenius B-modules (or simply $F$-modules over $B$ ) as follows:

- An object of $\operatorname{FMod}(B)$ is a pair $\underline{M}=(M, F)$ consisting of a $B$-module $M$ which is locally free of finite rank, together with an injective $B$-linear map $F=$ $F_{M}: \sigma^{*} M \rightarrow M$, where $\sigma^{*} M:=M \otimes_{B, \sigma} B$.

- A morphism of Frobenius $B$-modules $\left(M, F_{M}\right) \rightarrow\left(N, F_{N}\right)$ is a $B$-linear map $\phi: M \rightarrow N$ between the underlying $B$-modules such that $\phi$ is $F$-equivariant, i.e. such that $\phi \circ F_{M}=F_{N} \circ \sigma^{*} \phi$. It is called an isomorphism if $\phi$ is an isomorphism of the underlying $B$-modules. 
Let $B^{\prime}$ be a flat $B$-algebra together with a ring endomorphism $\sigma: B^{\prime} \rightarrow$ $B^{\prime}$ extending the Frobenius lift of $B$, as explained before. Then the exact functor - $\otimes_{B} B^{\prime}$ from $B$-modules to $B^{\prime}$-modules yields a functor $\operatorname{FMod}(B) \rightarrow \operatorname{FMod}\left(B^{\prime}\right)$. If the structure map $B \rightarrow B^{\prime}$ is, in addition, injective then the induced functor on FMod $(B)$ is faithful since, given a map $f: M \rightarrow N$ of finite projective $B$-modules, restricting its image $f \otimes \mathrm{id}: M \otimes_{B} B^{\prime} \rightarrow N \otimes_{B} B^{\prime}$ to $M$ gives back $f$. In particular, we obtain a natural commutative diagram of categories and faithful functors

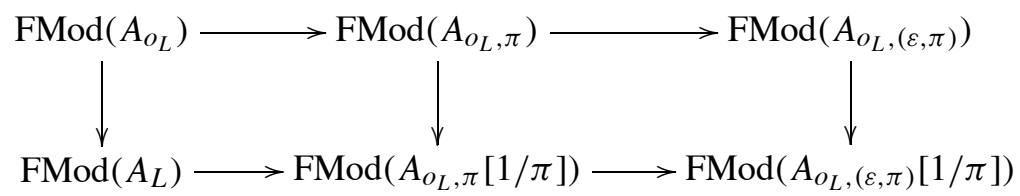

Slightly abusing notation, we agree to write $\underline{M} \otimes_{B} B^{\prime}$ for $\left(M \otimes_{B} B^{\prime}, F_{M} \otimes \mathrm{id}_{B^{\prime}}\right)$, whenever $\underline{M}=\left(M, F_{M}\right)$.

\section{Anderson motives}

Let $\mathfrak{J} \subset A_{o_{L}}$ be the ideal generated by $a \otimes 1-1 \otimes c^{*}(a)$ for all $a \in A$. For example, if $\mathcal{C}=\mathbb{P}_{\mathbb{F}}^{1}$ and $A=\mathbb{F}[z]$, then $\mathfrak{J}=(z-\zeta) \subset o_{L}[z]$ where $\zeta=c^{*}(z)$. Note that the convention introduced in Remark 2.1 that $(z)=\varepsilon^{m}$ implies $\zeta \in \mathfrak{m}_{L}$. So $\zeta=0$ if $c^{*}$ is not injective. By abuse of notation we denote the ideal generated by $\mathfrak{J}$ in any $A_{o_{L}}$-algebra again by $\mathfrak{J}$. We consider the following variant of Anderson's [1] $t$-motives.

Definition 4.1. An Anderson A-motive over $L$ is an object $\underline{M}=\left(M, F_{M}\right) \in$ $\operatorname{FMod}\left(A_{L}\right)$ such that $\operatorname{coker}\left(F_{M}\right)$ is a finite-dimensional $L$-vector space and is annihilated by a power of $\mathfrak{J}$. A morphism of Anderson $A$-motives is defined as a morphism inside $\operatorname{FMod}\left(A_{L}\right)$.

Since $\operatorname{Spec}\left(A_{L}\right)$ is of finite type over $L$, one can consider its rigid analytification $\operatorname{Spec}\left(A_{L}\right)^{\text {an }}$; see $[4,5,11]$. In accordance with [2], we denote this rigid analytic $L$-space by $\mathfrak{A}(\infty)$. On the other hand, the formal completion of the $o_{L^{-}}$ scheme $X=\operatorname{Spec}\left(A_{o_{L}}\right)$ along its special fiber $V(\pi)$ leads to the formal $o_{L}$-scheme $\mathfrak{X}=\operatorname{Spf}\left(A_{o_{L}, \pi}\right)$; see [14, $\left.\mathrm{I}_{\text {new }}, \mathrm{I} .10 .8 .3\right]$. Its associated rigid analytic space $\mathfrak{X}_{\text {rig }}$ $([4,11])$ is given by the affinoid $L$-space $\mathfrak{A}(1):=\operatorname{Sp}\left(A_{o_{L}, \pi}[1 / \pi]\right)$. This space can be regarded as the unit disc of the rigid analytic space $\mathfrak{A}(\infty)$ as it corresponds to "radius of convergence 1 ", hence the notation.

We study the following instance of rigid analytic $\tau$-sheaves over $A_{o_{L}, \pi}[1 / \pi]$, in the sense of [2].

Definition 4.2. An analytic Anderson A(1)-motive over $L$ is an object $\underline{M}=$ $\left(M, F_{M}\right) \in \operatorname{FMod}\left(A_{o_{L}, \pi}[1 / \pi]\right)$ such that $\operatorname{coker}\left(F_{M}\right)$ is a finite-dimensional $L$ vector space and is annihilated by a power of $\mathfrak{J}$. A morphism of analytic Anderson $A(1)$-motives is defined as a morphism in the category $\operatorname{FMod}\left(A_{o_{L}, \pi}[1 / \pi]\right)$. 
Here the prefix " $A(1)-$ " indicates that we are considering an analytic variant of Anderson A-motives over the rigid analytic "unit disc" $\mathfrak{A}(1)$ in $\operatorname{Spec}\left(A_{L}\right)$.

Proposition 4.3. The natural functor $\operatorname{FMod}\left(A_{L}\right) \rightarrow \operatorname{FMod}\left(A_{o_{L}, \pi}[1 / \pi]\right), \underline{M} \mapsto$ $\underline{M} \otimes_{A_{L}} A_{o_{L}, \pi}[1 / \pi]$ restricts to a functor (Anderson A-motives over $L$ ) $\rightarrow$ (analytic Anderson A(1)-motives over $L$ ).

Definition 4.4. (a) Let $\underline{M}_{L} \in \operatorname{FMod}\left(A_{L}\right)$ be an $F$-module over $A_{L}$. A model of $\underline{M}_{L}$ is a pair $(\underline{\mathcal{M}}, \alpha)$ consisting of an object $\underline{\mathcal{M}} \in \operatorname{FMod}\left(A_{O_{L}}\right)$ and an isomorphism $\alpha: \underline{M}_{L} \stackrel{\sim}{\longrightarrow} \underline{\mathcal{M}} \otimes_{A_{o_{L}}} A_{L}$ inside $\operatorname{FMod}\left(A_{L}\right)$.

(b) Let $\underline{M}_{L} \in \operatorname{FMod}\left(A_{o_{L}, \pi}[1 / \pi]\right)$ be an $F$-module over $A_{o_{L}, \pi}[1 / \pi]$. A (formal) model of $\underline{M}_{L}$ is a pair $(\mathcal{M}, \alpha)$ consisting of an object $\mathcal{M} \in \operatorname{FMod}\left(A_{o_{L}, \pi}\right)$ and an isomorphism $\alpha: \underline{M}_{L} \stackrel{\sim}{\longrightarrow} \underline{\mathcal{M}} \otimes_{A_{o_{L}, \pi}} A_{o_{L}, \pi}[1 / \pi]$ inside $\operatorname{FMod}\left(A_{o_{L}, \pi}[1 / \pi]\right)$.

(c) In both cases a morphism of models $\beta:(\underline{\mathcal{M}}, \alpha) \rightarrow\left(\underline{\mathcal{M}^{\prime}}, \alpha^{\prime}\right)$ is an isomorphism $\beta: \underline{\mathcal{M}} \stackrel{\sim}{\longrightarrow} \underline{\mathcal{M}}^{\prime}$ of $F$-modules satisfying $\alpha^{\prime}=\beta[1 / \pi] \circ \alpha$. In particular the sets $\operatorname{Hom}\left((\underline{\mathcal{M}}, \alpha),\left(\underline{\mathcal{M}}^{\prime}, \alpha^{\prime}\right)\right)$ contain at most one element.

We will sometimes drop the $\alpha$ from the notation and simply speak of $\underline{\mathcal{M}}$ as a model of $\underline{M}_{L}$.

For every $\underline{\mathcal{M}} \in \operatorname{FMod}\left(A_{o_{L}}\right)$, respectively $\underline{\mathcal{M}} \in \operatorname{FMod}\left(A_{o_{L}, \pi}\right)$ we can consider the reduction $\underline{\mathcal{M}} \otimes_{A_{o_{L}}} A_{\ell}$, respectively $\underline{\mathcal{M}} \otimes_{A_{o_{L}, \pi}} A_{\ell}$. Note, however, that this does not induce a functor from $\operatorname{FMod}\left(A_{O_{L}}\right)$, respectively $\operatorname{FMod}\left(A_{O_{L}, \pi}\right)$ to $\operatorname{FMod}\left(A_{\ell}\right)$, since the induced $F$-map need not be injective. This circumstance lies at the origin of our study of good models:

Definition 4.5. Let $\mathcal{M}$ be a model of an $F$-module $\underline{M}_{L}$ over $A_{L}$, respectively over $A_{o_{L}, \pi}[1 / \pi]$. Then $\underline{\mathcal{M}}$ is called a good model if $\underline{\mathcal{M}} / \pi \underline{\mathcal{M}}$ is an $F$-module over $A_{\ell}$, i.e. if the induced $A_{\ell}$-linear map

$$
\bar{\sigma}^{*}(\mathcal{M} / \pi \mathcal{M})=(\mathcal{M} / \pi \mathcal{M}) \otimes_{A_{\ell}, \bar{\sigma}} A_{\ell} \rightarrow \mathcal{M} / \pi \mathcal{M}
$$

is injective.

If $\underline{M}_{L}$ is an (analytic) Anderson motive there is an alternative notion of good reduction as follows.

Definition 4.6. Let $\underline{\mathcal{M}}$ be a model of an Anderson $A$-motive $\underline{M}_{L}$, respectively of an analytic Anderson $A(1)$-motive $\underline{M}_{L}$. Then $\underline{\mathcal{M}}$ is called a good model in the strong sense if $\operatorname{coker}\left(F_{\mathcal{M}}\right)$ is a finite free $o_{L}$-module and is annihilated by $\mathfrak{J}^{d}$, for some $d \geq 0$. In this case we also say that $\underline{\mathcal{M}}$ has good reduction over $o_{L}$.

Theorem 4.7. Let $\underline{\mathcal{M}}$ be a model of an Anderson A-motive, respectively of an analytic Anderson A(1)-motive $\underline{M}_{L}$. Then $\mathcal{M}$ is a good model in the weak sense of Definition 4.5 if and only if it is a good model in the strong sense of Definition 4.6.

Proof. Since $\sigma^{*} \mathcal{M}$ is locally free over $A_{o_{L}}$, respectively over $A_{o_{L}, \pi}$, the natural map $\sigma^{*} \mathcal{M} \rightarrow \sigma^{*} M_{L}$ is injective and hence $F_{\mathcal{M}}$ is injective because $F_{M_{L}}$ is. We obtain a short eqact sequence

$$
0 \longrightarrow \sigma^{*} \mathcal{M} \stackrel{F_{\mathcal{M}}}{\longrightarrow} \mathcal{M} \longrightarrow \operatorname{coker}\left(F_{\mathcal{M}}\right) \longrightarrow 0 .
$$


Let $\underline{\mathcal{M}}$ be a good model in the strong sense. Tensoring the short exact sequence (4.1) with $\ell$ over $o_{L}$ and using that $\operatorname{coker}\left(F_{\mathcal{M}}\right)$ is supposed to be free over $o_{L}$ shows that the induced $A_{\ell}$-linear map $\bar{\sigma}^{*}(\mathcal{M} / \pi \mathcal{M}) \rightarrow \mathcal{M} / \pi \mathcal{M}$ remains injective. So $\mathcal{M}$ is a good model in the weak sense.

Conversely suppose that $\underline{\mathcal{M}}$ is a good model in the weak sense. This time tensoring (4.1) with $\ell$ over $o_{L}$ yields

$$
\begin{aligned}
0 \longrightarrow \operatorname{Tor}_{1}^{o_{L}}\left(\operatorname{coker} F_{\mathcal{M}}, \ell\right) & \longrightarrow \sigma^{*} \mathcal{M} \otimes_{o_{L}} \ell \stackrel{F_{\mathcal{M}} \otimes \mathrm{id}_{\ell}}{\longrightarrow} \mathcal{M} \otimes_{o_{L}} \ell \\
& \longrightarrow \operatorname{coker}\left(F_{\mathcal{M}}\right) \otimes_{o_{L}} \ell \longrightarrow 0
\end{aligned}
$$

By assumption $F_{\mathcal{M}} \otimes \mathrm{id}_{\ell}$ is injective, and so $0=\operatorname{Tor}_{1}^{o_{L}}\left(\operatorname{coker} F_{\mathcal{M}}, \ell\right)=\{x \in$ $\left.\operatorname{coker}\left(F_{\mathcal{M}}\right): \pi x=0\right\}$ and $\operatorname{coker}\left(F_{\mathcal{M}}\right)$ is flat over $o_{L}$ by [10, Corollary 6.3]. This implies $\operatorname{coker}\left(F_{\mathcal{M}}\right) \hookrightarrow \operatorname{coker}\left(F_{\mathcal{M}}\right) \otimes_{o_{L}} L=\operatorname{coker}\left(F_{M_{L}}\right)$. Since $\operatorname{coker}\left(F_{M_{L}}\right)$ is annihilated by $\mathfrak{J}^{d}$ for some $d$, the same is true for $\operatorname{coker}\left(F_{\mathcal{M}}\right)$ which therefore is a finitely generated $A_{o_{L}} / \mathfrak{J}^{d}$-module, respectively $A_{o_{L}, \pi} / \mathfrak{J}^{d}$-module, and a fortiori a finitely generated $o_{L}$-module. Being flat, $\operatorname{coker}\left(F_{\mathcal{M}}\right)$ is a finite free $o_{L}$-module. Thus $\mathcal{M}$ is a good model in the strong sense.

Remark 4.8. In [13] Gardeyn develops a theory of semi-stable reduction of analytic Anderson $A(1)$-motives $\underline{M}_{L}$. He shows that after replacing $L$ by a finite separable extension, $\underline{M}_{L}$ has a model $\underline{\mathcal{M}}$ such that the reduction $F_{\mathcal{M}} \otimes \mathrm{id}_{\ell}$ is not nilpotent [13, Proposition 3.3]. If $\underline{\mathcal{M}}^{\prime} \subset \underline{\mathcal{M}} / \pi \underline{\mathcal{M}}$ is the maximal Frobenius $A_{\ell-}$ submodule with injective $F_{\overline{\mathcal{M}}^{\prime}}$, he further shows that the support of $\operatorname{coker}\left(F_{\overline{\mathcal{M}}^{\prime}}\right)$ is a finite set $S \subset$ Spec $A_{\ell}$. After removing $S$ from $\mathfrak{A}(1):=\operatorname{Sp}\left(A_{o_{L}, \pi}[1 / \pi]\right)$ one can lift $\underline{\overline{\mathcal{M}}^{\prime}}$ to an $F$-submodule $\left.\underline{\mathcal{M}}^{\prime} \subset \underline{\mathcal{M}}\right|_{\mathfrak{A}(1) \backslash S}$ which has good reduction in the weak sense of Definition 4.5; see [13, Theorem 4.7]. As one sees from the following example, it is false in general that $S$ is the zero locus of $\mathfrak{J}$ in $\operatorname{Spec} A_{\ell}$ and so we cannot expect that $\underline{\mathcal{M}}^{\prime}$ has good reduction in the strong sense of Definition 4.6.

Let $A=\mathbb{F}[z]$ and $\zeta=c^{*}(z) \in \mathfrak{m}_{L}$. Then $\mathfrak{J}=(z-\zeta)$. Let $\mathcal{M}=o_{L}\langle z\rangle{ }^{\oplus 2}$ and $F_{\mathcal{M}}=\left(\begin{array}{cc}0 & \pi(z-\zeta) \\ \pi & z-1\end{array}\right)$. Then $\underline{\mathcal{M}}=\left(\mathcal{M}, F_{\mathcal{M}}\right)$ is a model of the analytic Anderson $A(1)$-motive $\underline{M}_{L}:=\underline{\mathcal{M}} \otimes_{o_{L}} L$. The reduction $\underline{\mathcal{M}} / \pi \underline{\mathcal{M}}=\left(\ell[z]^{\oplus 2},\left(\begin{array}{ll}0 & 0 \\ 0 & z-1\end{array}\right)\right)$ contains the maximal Frobenius $A_{\ell}$-submodule $\underline{\overline{\mathcal{M}}^{\prime}}=\ell[z] \cdot\left(\begin{array}{l}0 \\ 1\end{array}\right)$, whose Frobenius is $F_{\overline{\mathcal{M}}^{\prime}}=z-1$. So $S=V(z-1) \neq V(z)=V(\mathfrak{J})$.

Proposition 4.9. If $\underline{M}_{L}$ is an Anderson A-Motive over L having a (good) model $\underline{\mathcal{M}}$ then its analytification $M_{L} \otimes_{A_{L}} A_{o_{L}, \pi}[1 / \pi]$ is an analytic Anderson A(1)-motive having the (good) model $\underline{\widehat{\mathcal{M}}}:=\underline{\mathcal{M}} \otimes_{A_{o_{L}}} A_{o_{L}, \pi}$ and the reduction $\underline{\widehat{\mathcal{M}}} / \pi \underline{\widehat{\mathcal{M}}}$ of $\underline{\widehat{\mathcal{M}}}$ is canonically isomorphic to the reduction $\underline{\mathcal{M}} / \pi \underline{\mathcal{M}}$ of $\underline{\mathcal{M}}$.

Proof. The statement without the properties of being a good model is obvious. From the isomorphism $\underline{\mathcal{M}} / \pi \underline{\mathcal{M}} \stackrel{\sim}{\longrightarrow} \underline{\mathcal{M}} / \pi \underline{\mathcal{M}}$ it follows that $\underline{\mathcal{M}}$ is a good model in the sense of Definition 4.5 if and only if $\widehat{\widehat{\mathcal{M}}}$ is a good model in the sense of Definition 4.5 . 
Let us also mention the following result of Gardeyn on good reduction of Drinfeld $A$-modules.

Proposition 4.10. Let $\phi: A \rightarrow L[\tau]$ be a Drinfeld A-module over L; see [9] or [21]. Let $M=M(\phi)$ be the associated Anderson A-motive; see [1, Section 4.1] or [12, Section 8.1]. Then the following are equivalent:

(i) $\phi$ has good reduction over $o_{L}$, i.e. $\phi$ is isomorphic over $L$ to a Drinfeld $A$ module $\psi: A \rightarrow L[\tau]$ satisfying $\psi(A) \subset o_{L}[\tau]$ such that the reduction $\bar{\psi}: A \rightarrow o_{L}[\tau] \rightarrow \ell[\tau]$ is a Drinfeld A-module over $\ell$ of the same rank as $\psi$ and $\phi$

(ii) $\underline{M}$ has good reduction over $o_{L}$ in the weak and strong senses of Definitions 4.6 and 4.5.

Proof. Gardeyn [12, Theorem 8.1] proved that $\phi$ has good reduction over $o_{L}$ if and only if $\underline{M}$ has a good model in the weak sense. So the proposition follows from Theorem 4.7 .

\section{Local shtukas and analytic Anderson motives}

Anderson A-motives can be viewed as function-field analogs of Abelian varieties. Barsotti-Tate groups, which can be associated with Abelian varieties over $\mathbb{Z}_{p^{-}}$ schemes, have effective local shtukas as function-field analogs.

Definition 5.1. An (effective) local shtuka at $\varepsilon$ over $o_{L}$ is an object $\underline{\hat{M}}=\left(\hat{M}, F_{\hat{M}}\right) \in$ $\operatorname{FMod}\left(A_{o_{L},(\varepsilon, \pi)}\right)$ such that $\operatorname{coker}\left(F_{\hat{M}}\right)$ is a finite free $o_{L}$-module and is annihilated by a power of $\mathfrak{J}$.

Remark 5.2. If the residue field $\mathbb{F}_{\varepsilon}=A / \varepsilon$ of $\varepsilon$ is larger than $\mathbb{F}$, i.e., if the degree $d_{\varepsilon}:=\left[\mathbb{F}_{\varepsilon}: \mathbb{F}\right]>1$, the ring $A_{o_{L},(\varepsilon, \pi)}$ is not an integral domain but a product $A_{o_{L},(\varepsilon, \pi)}=\prod_{i \in \mathbb{Z} / d_{\varepsilon} \mathbb{Z}} A_{o_{L},(\varepsilon, \pi)} / \mathfrak{a}_{i}$ of integral domains. To describe this product decomposition, note that $A_{o_{L},(\varepsilon, \pi)}=\lim _{n} A_{o_{L}} / \varepsilon^{n}=\lim _{n}\left(A / \varepsilon^{n}\right) \otimes_{\mathbb{F}} o_{L}=A_{\varepsilon} \widehat{\otimes}_{\mathbb{F}} o_{L}$. By Cohen's structure theorem $A_{\varepsilon} \cong \mathbb{F}_{\varepsilon} \llbracket z_{\varepsilon} \rrbracket$ for a uniformizer $z_{\varepsilon}$ of $A$ at $\varepsilon$. Then $\mathfrak{a}_{i}=\left(\alpha \otimes 1-1 \otimes c^{*}(\alpha)^{r^{i}}: \alpha \in \mathbb{F}_{\varepsilon} \subset A_{\varepsilon}\right)$, where we use that $c^{*}: A \rightarrow o_{L}$ factors through $c^{*}: A_{\varepsilon} \rightarrow o_{L}$. The factors $A_{o_{L},(\varepsilon, \pi)} / \mathfrak{a}_{i}$ are isomorphic to $o_{L} \llbracket z_{\varepsilon} \rrbracket$ and hence are integral domains. They are cyclically permuted by $\sigma$ because $\sigma\left(\mathfrak{a}_{i}\right)=$ $\mathfrak{a}_{i+1}$. By [3, Proposition 8.8] the functor $\left(\hat{M}, F_{\hat{M}}\right) \mapsto\left(\hat{M} / \mathfrak{a}_{0} \hat{M},\left(F_{\hat{M}}\right)^{d_{\varepsilon}}\right)$ is an equivalence between the category of effective local shtukas at $\varepsilon$ over $o_{L}$ as in Definition 5.1 and the category of pairs $\left(\hat{M}_{0}, \widetilde{F}_{\hat{M}}\right)$ where $\hat{M}_{0}$ is a free module of finite rank over $A_{o_{L},(\varepsilon, \pi)} / \mathfrak{a}_{0}$ and $\widetilde{F}_{\hat{M}}:\left(\sigma^{d_{\varepsilon}}\right)^{*} \hat{M}_{0} \rightarrow \hat{M}_{0}$ is injective with coker $\left(\widetilde{F}_{\hat{M}}\right)$ being a finite free $o_{L}$-module. In $[16,17]$ these pairs $\left(\hat{M}_{0}, \widetilde{F}_{\hat{M}}\right)$ are called (effective) local shtukas.

The following criterion for good reduction of analytic Anderson A(1)-motives can be regarded as a good-reduction Local-Global Principle at the characteristic place. 
Theorem 5.3. Let $\underline{M}_{L}=\left(M_{L}, F_{M_{L}}\right)$ be an analytic Anderson A(1)-motive over $L$ such that $\operatorname{coker}\left(F_{M_{L}}\right)$ is annihilated by $\mathfrak{J}^{d}$ for some $d$. Then the following assertions are equivalent:

(i) $\underline{M}_{L}$ admits a good model in the strong sense of Definition 4.6;

(ii) There is an effective local shtuka $\underline{\hat{M}}=\left(\hat{M}, F_{\hat{M}}\right)$ at $\varepsilon$ over $o_{L}$ such that $\operatorname{coker}\left(F_{\hat{M}}\right)$ is annihilated by $\mathfrak{J}^{d}$, and an isomorphism $\underline{M}_{L} \otimes_{A_{o_{L}, \pi}[1 / \pi]} A_{o_{L},(\varepsilon, \pi)}[1 / \pi] \cong$ $\underline{\hat{M}} \otimes_{A_{o_{L},(\varepsilon, \pi)}} A_{o_{L},(\varepsilon, \pi)}[1 / \pi]$ in $\operatorname{FMod}\left(A_{o_{L},(\varepsilon, \pi)}[1 / \pi]\right)$.

Proof. 1. In order to show that (ii) implies (i), we let $f: M_{L} \otimes A_{o_{L},(\varepsilon, \pi)}[1 / \pi] \stackrel{\sim}{\longrightarrow}$ $\hat{M} \otimes A_{o_{L},(\varepsilon, \pi)}[1 / \pi]=: \hat{M}[1 / \pi]$ be an $F$-equivariant isomorphism of $A_{o_{L},(\varepsilon, \pi)}[1 / \pi]$ modules as in (ii). We have canonical $F$-equivariant $A_{o_{L}, \pi}$-linear maps

$$
i: M_{L} \rightarrow M_{L} \otimes_{A_{o_{L}, \pi}[1 / \pi]} A_{o_{L},(\varepsilon, \pi)}[1 / \pi], \quad j: \hat{M} \rightarrow \hat{M}[1 / \pi]
$$

where $i$ (respectively, $j$ ) is injective since $M_{L}$ (respectively, $\hat{M}$ ) is flat. Consider the $A_{o_{L}, \pi}$-module $\mathcal{M}=\operatorname{im}(i) \cap f^{-1}(\operatorname{im}(j))$. We will show that $\mathcal{M}$ is a good model of $M_{L}$. The inclusion $\mathcal{M} \hookrightarrow M_{L}$ gives rise to an $A_{o_{L}, \pi}[1 / \pi]$-linear embedding $\mathcal{M}[1 / \pi] \hookrightarrow M_{L}[1 / \pi] \cong M_{L}$, which is in fact an isomorphism, because if $m \in M_{L}$ there is an $s \geq 0$ such that $\pi^{s} f(m \otimes 1) \in \operatorname{im}(j)$, i.e. $\pi^{s} m \in \mathcal{M}$.

2. In order to show that $\mathcal{M}$ is a finitely generated $A_{o_{L}, \pi}$-module we use the embed$\operatorname{ding} \iota: \mathbb{F}[z] \rightarrow A$ from Remark 2.1 and the induced maps $L\langle z\rangle \rightarrow A_{o_{L}, \pi}[1 / \pi]$ and $o_{L} \llbracket z \rrbracket \rightarrow A_{o_{L},(\varepsilon, \pi)}$ from (2.1). Let $\left(e_{1}, \ldots, e_{m}\right)$ be a basis of $M_{L}$ over the principal ideal domain $L\langle z\rangle$. Furthermore, let $\left(d_{1}, \ldots, d_{n}\right)$ be a basis for $\hat{M}$ over the local ring $o_{L} \llbracket z \rrbracket$. Note that the basis $\left(e_{1}, \ldots, e_{m}\right)$ gives rise to an isomorphism $M_{L} \otimes_{L\langle z\rangle} o_{L} \llbracket z \rrbracket[1 / \pi] \cong o_{L} \llbracket z \rrbracket[1 / \pi]^{\oplus m}$. For every $v=1, \ldots, n$ we consider $f^{-1}\left(d_{\nu}\right)$ and regard it as an element of the right-hand side of this isomorphism. We choose $N \geq 0$ big enough, such that $f^{-1}\left(\pi^{N} d_{\nu}\right) \in o_{L} \llbracket z \rrbracket^{\oplus m}$ for all $v$, say

$$
f^{-1}\left(\pi^{N} d_{v}\right)=\left(\rho_{v, 1}, \ldots, \rho_{v, m}\right)
$$

where $\rho_{v, \mu} \in o_{L} \llbracket z \rrbracket$. Now let $x \in \mathcal{M}$. Via $f$ we obtain $f(x)=\sum_{\nu} \lambda_{v} d_{v}$ in $\hat{M}$, with suitable $\lambda_{v} \in o_{L} \llbracket z \rrbracket$. Consequently $f\left(\pi^{N} x\right)=\sum_{v} \lambda_{v}\left(\pi^{N} d_{v}\right)$, so that the image of $\pi^{N} x$ in $o_{L} \llbracket z \rrbracket^{\oplus m}$ satisfies $\pi^{N} x=\sum_{\mu}\left(\sum_{\nu} \lambda_{\nu} \rho_{\nu, \mu}\right) e_{\mu}$. The appearing scalars $h_{\mu}=\sum_{v} \lambda_{\nu} \rho_{v, \mu}$ have, in fact, to be elements of $L\langle z\rangle \cap o_{L} \llbracket z \rrbracket=o_{L}\langle z\rangle$. Inside $M_{L}$ we may write $x=\pi^{-N} \pi^{N} x=\sum_{\mu} h_{\mu} \pi^{-N} e_{\mu}$, so that we may conclude

$$
\mathcal{M} \subset \sum_{\mu} o_{L}\langle z\rangle \pi^{-N} e_{\mu} .
$$

Being a submodule of a finitely generated module over a noetherian ring, $\mathcal{M}$ has to be a finitely generated $o_{L}\langle z\rangle$-module and hence a finitely generated $A_{o_{L}, \pi}$-module. 3. We claim that $\mathcal{M} / \pi \mathcal{M}$ is torsion-free and hence free over $\ell[z]$, because it is finitely generated. Let $x \in \mathcal{M}$, and let $\lambda \in o_{L}\langle z\rangle$ be such that $\lambda \notin \pi o_{L}\langle z\rangle$ and $\lambda x \in$ 
$\pi \mathcal{M}$, say $\lambda x=\pi y$ for some $y \in \mathcal{M}$. In order to prove that $\mathcal{M} / \pi \mathcal{M}$ is torsion-free we must show that $x \in \pi \mathcal{M}$. First suppose that $\lambda \in o_{L}\langle z\rangle \cap o_{L} \llbracket z \rrbracket^{\times}$. We consider $\pi^{-1} x \in M_{L}$. In fact, this element lies in $\mathcal{M}$, since we have $f\left(\pi^{-1} x\right)=\lambda^{-1} f(y) \in$ $\hat{M}$. Consequently $x=\pi\left(\pi^{-1} x\right) \in \pi \mathcal{M}$.

Let us next assume that $\lambda=z^{n}$ and show that $z^{n} x \in \pi \mathcal{M}$ implies $x \in \pi \mathcal{M}$ for any $n \geq 0$. By induction, it suffices to consider the case $n=1$. So suppose $z x \in \pi \mathcal{M}$, say $z x=\pi y$. Let $f(x)=\sum_{v} \beta_{\nu} d_{\nu}$, where $\left(d_{1}, \ldots, d_{n}\right)$ is the finite $o_{L} \llbracket z \rrbracket$-basis of $\hat{M}$ fixed before. The relation $z x=\pi y$ implies that $\pi \mid z \beta_{v}$ for every index $v$, so that $\pi \mid \beta_{v}$ for every $v$. Therefore $\pi^{-1} x \in M_{L}$ necessarily maps via $f$ to an element of $\hat{M}$, i.e. $x \in \pi \mathcal{M}$.

Finally we treat the case for general $\lambda=\sum_{s} \lambda_{s} z^{s}$ and suppose that $\lambda \notin$ $o_{L} \llbracket z \rrbracket^{\times}$, that is $\pi \mid \lambda_{0}$. This means we find $\lambda^{\prime} \in o_{L}[z]$ and $\lambda^{\prime \prime} \in o_{L}\langle z\rangle \cap o_{L} \llbracket z \rrbracket^{\times}$ such that $\lambda=\pi \lambda^{\prime}+z^{N} \lambda^{\prime \prime}$ for some $N \geq 1$. We have $\pi y=\lambda x=\pi \lambda^{\prime} x+z^{N} \lambda^{\prime \prime} x$. In particular $z^{N} \lambda^{\prime \prime} x=\pi\left(y-\lambda^{\prime} x\right) \in \pi \mathcal{M}$ and by the above $\lambda^{\prime \prime} x \in \pi \mathcal{M}$ and $x \in \pi \mathcal{M}$.

Thus we have proved that $\mathcal{M} / \pi \mathcal{M}$ is free over $\ell[z]$. It follows that $\mathcal{M} / \pi \mathcal{M}$ is locally free of finite rank over $A_{\ell}$.

4. We claim that $\mathcal{M}$ is locally free of finite rank over $A_{o_{L}, \pi}$. Since it is finitely generated it only remains to show that $\mathcal{M}$ is flat over $A_{o_{L}, \pi}$. Since $A_{o_{L}, \pi}$ is $\pi$-adically complete and separated, $\pi A_{o_{L}, \pi}$ is contained in the Jacobson radical $\mathrm{j}\left(A_{o_{L}, \pi}\right)$ by [20, Theorem 8.2], and the $A_{o_{L}, \pi}$-module $\mathcal{M}$ is finitely generated, so that $\mathcal{M}$ is $\pi$-adically ideally Hausdorff in the sense of [8, III.5.1]. In the preceding step we have shown that $\mathcal{M} / \pi \mathcal{M}$ is flat over $A_{\ell} \cong A_{o_{L}, \pi} / \pi A_{o_{L}, \pi}$, and we know that $\mathcal{M}$ has no $\pi$-torsion, so that the canonical map $\pi A_{o_{L}, \pi} \otimes_{A_{o_{L}, \pi}} \mathcal{M} \rightarrow \pi \mathcal{M}$ is an isomorphism. Therefore, by Bourbaki's Flatness Criterion [8, Section III.5.2, Théorème 1(iii)], we may conclude that $\mathcal{M}$ is indeed flat over $A_{o_{L}, \pi}$.

5. We note that $\sigma^{*} \mathcal{M}=\sigma^{*} \operatorname{im}(i) \cap\left(\sigma^{*} f\right)^{-1}\left(\sigma^{*} \operatorname{im}(j)\right)$ because the functor $\sigma^{*}$ is exact by Lemma 3.1. By the $F$-equivariance of $f$ we obtain a Frobenius $F_{\mathcal{M}}$ : $\sigma^{*} \mathcal{M} \rightarrow \mathcal{M}$. It is injective because $F_{M_{L}}$ is. We set $\underline{\mathcal{M}}:=\left(\mathcal{M}, F_{\mathcal{M}}\right)$.

6. Next we claim that $\mathfrak{J}^{d} \operatorname{coker}\left(F_{\mathcal{M}}\right)=0$. Let $x=\sum_{v} h_{v} m_{v} \in \mathfrak{J}^{d} \mathcal{M}$ where $h_{v} \in \mathfrak{J}^{d}$ and $m_{v} \in \mathcal{M}$. Since $\operatorname{coker}\left(F_{M_{L}}\right)$ is annihilated by $\mathfrak{J}^{d}$, there is a (unique) $y \in \sigma^{*} M_{L}$ such that $x=\sum_{\nu} h_{\nu} m_{v}=F_{M_{L}}(y)$. We have to show that $y \in \sigma^{*} \mathcal{M}=$ $\sigma^{*} \operatorname{im}(i) \cap\left(\sigma^{*} f\right)^{-1}\left(\sigma^{*} \operatorname{im}(j)\right)$. So it remains to see that $\left(\sigma^{*} f\right)(y) \in \operatorname{im}\left(\sigma^{*} j\right)$. Indeed, inside $\hat{M}[1 / \pi]$ we have $f(x)=f\left(F_{M_{L}}(y)\right)=F_{\hat{M}}\left(\left(\sigma^{*} f\right)(y)\right)$. On the other hand, the linearity of $f$ and $j$ gives that $f(x)=\sum_{v} h_{v} f\left(m_{v} \otimes 1\right)=j\left(y^{\prime}\right)$ for some $y^{\prime} \in \mathfrak{J}^{d} \hat{M} \subset \operatorname{im}\left(F_{\hat{M}}\right)$, say $y^{\prime}=F_{\hat{M}}\left(y^{\prime \prime}\right)$ for a $y^{\prime \prime} \in \sigma^{*} \hat{M}$. Thus $f(x)=$ $F_{\hat{M}}\left(\left(\sigma^{*} j\right)\left(y^{\prime \prime}\right)\right)$. So finally, since $F_{\hat{M}}: \sigma^{*} \hat{M}[1 / \pi] \rightarrow \hat{M}[1 / \pi]$ is injective, we obtain that $\left(\sigma^{*} f\right)(y)=\left(\sigma^{*} j\right)\left(y^{\prime \prime}\right)$, as desired.

7. Finally we show that the kernel $V$ of $\bar{F}: \sigma^{*}(\mathcal{M} / \pi \mathcal{M}) \rightarrow \mathcal{M} / \pi \mathcal{M}$ is trivial. This implies that $\underline{\mathcal{M}}$ is a good model of $\underline{M}_{L}$ in the weak sense of Definition 4.5 , which is enough by Theorem 4.7.

We have already shown that $\mathfrak{J}^{d} \mathcal{M} \subset \operatorname{im}\left(F_{\mathcal{M}}\right)$. Since $(z-\zeta) \in \mathfrak{J}$ for $\zeta:=$ $c^{*}(z) \in o_{L}$ we have a chain of $o_{L}\langle z\rangle$-modules $(z-\zeta)^{d} \mathcal{M} \subset \operatorname{im}\left(F_{\mathcal{M}}\right) \subset \mathcal{M}$. The 
element $\zeta \in o_{L}$ is zero $\bmod \pi$, and we obtain

$$
z^{d}(\mathcal{M} / \pi \mathcal{M}) \subset \operatorname{im}(\bar{F}) \subset \mathcal{M} / \pi \mathcal{M} .
$$

We know that $\mathcal{M} / \pi \mathcal{M}$ is finite free over $\ell[z]$. Therefore the middle term $W:=$ $\operatorname{im}(\bar{F})$ in the latter chain has full rank inside $\mathcal{M} / \pi \mathcal{M}$. Finally, taking ranks in the (split) short exact sequence of finite free $\ell[z]$-modules

$$
0 \rightarrow V \rightarrow \sigma^{*}(\mathcal{M} / \pi \mathcal{M}) \stackrel{\bar{F}}{\rightarrow} W \rightarrow 0
$$

accomplishes the proof that $V$ indeed is trivial.

8. Conversely, in order to show that (i) implies (ii), suppose that $(\underline{\mathcal{M}}, \alpha)$ is a good model of $\underline{M}_{L}$. We define

$$
\underline{\hat{M}}=\underline{\mathcal{M}} \otimes_{A_{o_{L}, \pi}} A_{o_{L},(\varepsilon, \pi)},
$$

i.e. $\underline{\hat{M}}$ equals the completion of $\underline{\mathcal{M}}$ for the $(\varepsilon, \pi) A_{o_{L}, \pi}$-adic topology. It is clear that the $F$-equivariant isomorphism $\alpha: M_{L} \stackrel{\sim}{\longrightarrow} \mathcal{M}[1 / \pi]$ of $A_{o_{L}, \pi}[1 / \pi]$-modules gives rise to a natural $F$-equivariant $A_{o_{L},(\varepsilon, \pi)}[1 / \pi]$-linear isomorphism

$$
M_{L} \otimes_{A_{o_{L}, \pi}[1 / \pi]} A_{o_{L},(\varepsilon, \pi)}[1 / \pi] \cong \hat{M}[1 / \pi] .
$$

We claim that $\hat{\underline{M}}$ is a local shtuka. Indeed, by base change, $\hat{M}$ is again locally free of finite rank. Furthermore, since the completion map $A_{o_{L}, \pi} \rightarrow A_{o_{L},(\varepsilon, \pi)}$ is Frobeniusequivariant and flat, we obtain an injective map $\hat{M} \otimes_{\left(A_{o_{L},(\varepsilon, \pi)}\right), \sigma} A_{o_{L},(\varepsilon, \pi)} \rightarrow \hat{M}$. Let $C^{\prime}$ be its cokernel, and let $C=\operatorname{coker}\left(F_{\mathcal{M}}\right)$, i.e. $C^{\prime} \cong C \otimes_{A_{o_{L}, \pi}} A_{o_{L},(\varepsilon, \pi)}$. Since $C$ is annihilated by $\mathfrak{J}^{d}$ the module $C^{\prime}$ equals $C$ and it is finite free over $o_{L}$. Thus $\underline{\hat{M}}$ is an effective local shtuka over $o_{L}$.

Remark 5.4. Steps 1-4 in the previous proof suggest that there is an equivalence of categories

$$
\begin{aligned}
& \mathcal{F}:\left\{\begin{array}{l}
\text { finite locally free } \\
A_{o_{L}, \pi} \text {-modules } \mathcal{M}
\end{array}\right\} \\
& \stackrel{\sim}{\longleftrightarrow}\left\{\begin{array}{l}
\text { triples }\left(M_{L}, \hat{M}, f\right) \text { consisting of } \\
\bullet \\
\bullet \text { a finite locally free } A_{o_{L}, \pi}[1 / \pi] \text {-module } M_{L}, \\
\bullet \text { an isomocally free } A_{o_{L},(\varepsilon, \pi)} \text {-module } \hat{M}, \text { and } \\
\quad f: M_{L} \otimes_{A_{o_{L}, \pi}[1 / \pi]} A_{o_{L},(\varepsilon, \pi)}[1 / \pi] \stackrel{\sim}{\longrightarrow} \hat{M} \otimes_{A_{o_{L},(\varepsilon, \pi)}} A_{o_{L},(\varepsilon, \pi)}[1 / \pi]
\end{array}\right\} \\
& \mathcal{M} \longmapsto\left(\mathcal{M} \otimes_{A_{o_{L}, \pi}} A_{o_{L}, \pi}[1 / \pi], \mathcal{M} \otimes_{A_{o_{L}, \pi}} A_{o_{L},(\varepsilon, \pi)}, \operatorname{id}_{\mathcal{M} \otimes A_{o_{L},(\varepsilon, \pi)}[1 / \pi]}\right),
\end{aligned}
$$


where on the right a morphism $\underline{h}=\left(h_{L}, \hat{h}\right):\left(M_{L}, \hat{M}, f\right) \rightarrow\left(M_{L}^{\prime}, \hat{M}^{\prime}, f^{\prime}\right)$ consists of a morphism $h_{L}: M_{L} \rightarrow M_{L}^{\prime}$ and a morphism $\hat{h}: \hat{M} \rightarrow \hat{M}^{\prime}$ such that $f^{\prime} \circ\left(h_{L} \otimes \operatorname{id}_{A_{o_{L},(\varepsilon, \pi)}[1 / \pi]}\right)=\left(\hat{h} \otimes \operatorname{id}_{A_{o_{L},(\varepsilon, \pi)}[1 / \pi]}\right) \circ f$.

However, this is false as can be seen from the following example, where we take $A=\mathbb{F}[z]$. We choose an element $a \in \ell \llbracket z \rrbracket \subset \ell((z))$ such that $a \notin \ell(z)$, and we let $\Delta=\left(\begin{array}{cc}1 & \pi^{-1} a \\ 0 & \pi^{-1}\end{array}\right)$. Set $M_{L}=L\langle z\rangle^{\oplus 2}, \hat{M}=\Delta \cdot o_{L} \llbracket z \rrbracket^{\oplus 2}$ and $f=\operatorname{id}_{o_{L} \llbracket z \rrbracket[1 / \pi]^{2}}$. Then $\Delta^{-1}=\left(\begin{array}{cc}1 & -a \\ 0 & \pi\end{array}\right) \in o_{L} \llbracket z \rrbracket^{2 \times 2}$ and

$$
o_{L} \llbracket z \rrbracket^{\oplus 2}=\Delta \cdot \Delta^{-1} o_{L} \llbracket z \rrbracket^{\oplus 2} \subset \hat{M} \subset \pi^{-1} o_{L} \llbracket z \rrbracket^{\oplus 2} .
$$

If there were a finite free $A_{o_{L}, \pi}$-module $\mathcal{M}$ with $\left(h_{L}, \hat{h}\right): \mathcal{F}(\mathcal{M}) \stackrel{\sim}{\longrightarrow}\left(M_{L}, \hat{M}, f\right)$, then it had to satisfy $\mathcal{M} \cong M_{L} \cap \hat{M}$ with $h_{L}$ and $\hat{h}$ induced from the inclusions $M_{L} \cap \hat{M} \subset M_{L}$ and $M_{L} \cap \hat{M} \subset \hat{M}$. So we may take directly $\mathcal{M}:=M_{L} \cap \hat{M}$. It satisfies $o_{L}\langle z\rangle^{\oplus 2} \subset \mathcal{M} \subset \pi^{-1} o_{L}\langle z\rangle^{\oplus 2}$. We claim that, in fact, the first inclusion is an equality. Namely let $\left(\begin{array}{c}v \\ w\end{array}\right)=\left(\begin{array}{c}\pi^{-1} v_{0}+v^{\prime} \\ \pi^{-1} w_{0}+w^{\prime}\end{array}\right) \in \mathcal{M}$ with $v_{0}, w_{0} \in \ell[z]$ and $v^{\prime}, w^{\prime} \in o_{L}\langle z\rangle$. Then $\Delta^{-1}\left(\begin{array}{c}v \\ w\end{array}\right)=\left(\begin{array}{c}\pi^{-1} v_{0}+v^{\prime}-\pi^{-1} a w_{0}-a w^{\prime} \\ w_{0}+\pi w^{\prime}\end{array}\right) \in o_{L} \llbracket z \rrbracket^{\oplus 2}$. This implies $v_{0}=a w_{0}$ in $\ell \llbracket z \rrbracket$. If $w_{0} \neq 0$ we get $a=v_{0} / w_{0} \in \ell(z)$ in contradiction to our assumption. So $w_{0}=v_{0}=0$ and $\left(\begin{array}{c}v \\ w\end{array}\right) \in o_{L}\langle z\rangle^{\oplus 2}$. This proves our claim that $\mathcal{M}=o_{L}\langle z\rangle^{\oplus 2}$. We conclude that $\mathcal{F}(\mathcal{M}) \supsetneqq\left(M_{L}, \hat{M}, f\right)$ and $\mathcal{F}$ is not an equivalence of categories.

After this example the following result is even more surprising.

Corollary 5.5. Let $\underline{M}_{L}$ be an analytic Anderson A(1)-motive over L. Then there is an equivalence of categories

$$
\begin{gathered}
\left\{\begin{array}{l}
\text { good models }(\underline{\mathcal{M}}, \alpha) \text { of } \underline{M}_{L} \text { in the } \\
\text { sense of Definitions } 4.6 \text { and } 4.5
\end{array}\right\} \\
\stackrel{\sim}{\longleftrightarrow}\left\{\begin{array}{c}
\text { pairs }(\hat{\underline{M}}, f) \text { consisting of } \\
\bullet \text { a local shtuka } \hat{\hat{M}} \text { at } \varepsilon \text { over } o_{L}, \text { and } \\
\bullet \text { an isomorphism in } \mathrm{FMod}\left(A_{o_{L},(\varepsilon, \pi)}[1 / \pi]\right) \\
f: \underline{M}_{L} \otimes A_{o_{L},(\varepsilon, \pi)}[1 / \pi] \stackrel{\sim}{\longrightarrow} \underline{\hat{M}}[1 / \pi]
\end{array}\right\} \\
(\underline{\mathcal{M}}, \alpha) \longmapsto(\underline{\mathcal{M}}, \alpha) \otimes_{A_{o_{L}, \pi}} A_{o_{L},(\varepsilon, \pi)},
\end{gathered}
$$

where on the right-hand side a morphism of pairs $\hat{\beta}:(\underline{\hat{M}}, f) \stackrel{\sim}{\longrightarrow}\left(\underline{\hat{M}}^{\prime}, f^{\prime}\right)$ is defined to be an isomorphism of local shtukas $\hat{\beta}: \underline{\hat{M}} \stackrel{\sim}{\longrightarrow} \underline{\hat{M}}^{\prime}$ satisfying $f^{\prime}=\hat{\beta} \circ f$. Proof. Suppose that $(\underline{\mathcal{M}}, \alpha)$ is a good model of $\underline{M}_{L}$. In the proof of 5.3 we have seen that its completion $\underline{\hat{\mathcal{M}}}:=\underline{\mathcal{M}} \otimes_{A_{o_{L}, \pi}} A_{o_{L},(\varepsilon, \pi)}$ is a local shtuka at $\varepsilon$. The $F$-equivariant isomorphism $\alpha: M_{L} \stackrel{\sim}{\longrightarrow} \mathcal{M}[1 / \pi]$ of $A_{o_{L}, \pi}[1 / \pi]$-modules induces 
the isomorphism

$$
\begin{aligned}
f & :=\alpha \otimes \operatorname{id}_{A_{o_{L},(\varepsilon, \pi)}[1 / \pi]}: M_{L} \otimes_{A_{o_{L}, \pi}[1 / \pi]} A_{o_{L},(\varepsilon, \pi)}[1 / \pi] \\
& \stackrel{\sim}{\longrightarrow} \hat{\mathcal{M}} \otimes_{A_{o_{L},(\varepsilon, \pi)}} A_{o_{L},(\varepsilon, \pi)}[1 / \pi]
\end{aligned}
$$

which is $F$-equivariant, and satisfies $\mathcal{M}=f\left(M_{L}\right) \cap \hat{\mathcal{M}}$, because $A_{o_{L}, \pi}=$ $A_{o_{L}, \pi}[1 / \pi] \cap A_{o_{L},(\varepsilon, \pi)}$.

To see that this functor is fully faithful let $(\underline{\mathcal{M}}, \alpha)$ and $\left(\underline{\mathcal{M}}^{\prime}, \alpha^{\prime}\right)$ be good models of $\underline{M}_{L}$ and let $\hat{\beta}:(\underline{\hat{\mathcal{M}}}, f):=(\underline{\mathcal{M}}, \alpha) \otimes_{A_{o_{L}, \pi}} A_{o_{L},(\varepsilon, \pi)} \stackrel{\sim}{\longrightarrow}\left(\underline{\hat{\mathcal{M}}^{\prime}}, f^{\prime}\right):=$ $\left(\underline{\mathcal{M}^{\prime}}, \alpha^{\prime}\right) \otimes_{A_{o_{L}, \pi}} A_{o_{L},(\varepsilon, \pi)}$ be an isomorphism. This means $f^{\prime}=\hat{\beta} \circ f$. Applying $\mathcal{M}=f\left(M_{L}\right) \cap \hat{\mathcal{M}}$ and $\mathcal{M}^{\prime}=f^{\prime}\left(M_{L}\right) \cap \hat{\mathcal{M}}^{\prime}$ we see that $\hat{\beta}(\mathcal{M})=\mathcal{M}^{\prime}$. Therefore $\beta:=\left.\hat{\beta}\right|_{\mathcal{M}}: \mathcal{M} \stackrel{\sim}{\longrightarrow} \mathcal{M}^{\prime}$ is the desired isomorphism satisfying $\beta \otimes \operatorname{id}_{A_{o_{L},(\varepsilon, \pi)}}=\hat{\beta}$. This implies $\alpha^{\prime}=\beta \circ \alpha$ and the $F$-equivariance of $\beta$, and hence $\beta:(\underline{\mathcal{M}}, \alpha) \stackrel{\sim}{\longrightarrow}$ $\left(\underline{\mathcal{M}}^{\prime}, \alpha^{\prime}\right)$.

To prove essential surjectivity, let a local shtuka $\underline{\hat{M}}$ together with an isomorphism $f: \underline{M}_{L} \otimes_{A_{o_{L}, \pi}[1 / \pi]} A_{o_{L},(\varepsilon, \pi)}[1 / \pi] \stackrel{\sim}{\sim} \underline{\hat{M}}[1 / \pi]$ be given. It remains to show that the $(\varepsilon, \pi) A_{o_{L}, \pi}$-adic completion $\underline{\hat{\mathcal{M}}}:=\underline{\mathcal{M}} \otimes_{A_{o_{L}, \pi}} A_{O_{L},(\varepsilon, \pi)}$ of the good model $\mathcal{M}=M_{L} \cap f^{-1}(\hat{M})$ gained in the proof of 5.3 gives back $\underline{\hat{M}}$. Then we take $\alpha$ as the canonical isomorphism id : $\mathcal{M} \otimes_{A_{o_{L}, \pi}} A_{o_{L}, \pi}[1 / \pi] \stackrel{\sim}{\longrightarrow} M_{L}$. By construction of $\underline{\mathcal{M}}$, the map $f$ restricts to an embedding $\mathcal{M} \hookrightarrow \hat{M}$, which in turn induces an $F$-equivariant and $A_{o_{L},(\varepsilon, \pi)}$-linear map $\psi:=\left.f\right|_{\hat{\mathcal{M}}}: \hat{\mathcal{M}} \rightarrow \hat{M}$, which becomes an isomorphism after inverting $\pi$. Our aim is to show that already the map $\psi$ is an isomorphism $(\underline{\mathcal{M}}, \mathrm{id}) \otimes_{A_{o_{L}, \pi}} A_{o_{L},(\varepsilon, \pi)} \stackrel{\sim}{\longrightarrow}(\underline{\hat{M}}, f)$. According to Remark 5.4 we have to use the Frobenius morphisms $F_{\hat{\mathcal{M}}}$ and $F_{\hat{M}}$ in an essential way.

We know that $\mathcal{M}$ is finite free over $o_{L}\langle z\rangle$ and that $\operatorname{rk}_{o_{L} \llbracket z \rrbracket}(\hat{\mathcal{M}})=\operatorname{rk}_{o_{L} \llbracket z \rrbracket}(\hat{M})=$ : $s$. We fix an $o_{L} \llbracket z \rrbracket$-basis $\mathfrak{B}$ (respectively, $\mathfrak{C}$ ) of $\hat{\mathcal{M}}$ (respectively, of $\hat{M}$ ) and let $\mathbf{A}=\mathfrak{C}[\psi]_{\mathfrak{B}} \in o_{L} \llbracket z \rrbracket^{s \times s}$ be the matrix which describes $\psi$ with respect to $\mathfrak{B}$ and $\mathfrak{C}$. Likewise, we let

$$
\mathbf{T}={ }_{\mathfrak{B}}\left[F_{\hat{\mathcal{M}}}\right]_{\sigma^{* \mathfrak{B}}}, \quad \mathbf{T}^{\prime}=\mathfrak{C}\left[F_{\hat{M}}\right]_{\sigma^{*} \mathfrak{C}}
$$

be the matrices corresponding to $F_{\hat{\mathcal{M}}}$ and $F_{\hat{M}}$, so that $\mathbf{A T}=\mathbf{T}^{\prime} \sigma(\mathbf{A})$ by virtue of the $F$-equivariance of $\psi$. In order to see that $\psi$ is an isomorphism, we need to show that $\operatorname{det}(\mathbf{A})$ is a unit in $o_{L} \llbracket z \rrbracket$. To begin with, an elementary application of the Weierstraß Division Theorem for $o_{L} \llbracket z \rrbracket([8$, VII.3.8.5]) shows that the kernel of the epimorphism $o_{L} \llbracket z \rrbracket \rightarrow o_{L}, z \mapsto \zeta$, is generated by $z-\zeta$, so that the latter is a prime element of $o_{L} \llbracket z \rrbracket$. Furthermore, recall that $o_{L} \llbracket z \rrbracket$, being a regular local ring, is factorial ( $[20], 20.3)$. We know that $\underline{\hat{\mathcal{M}}}$ is a local shtuka, so that $F_{\hat{\mathcal{M}}}$ becomes an isomorphism after inverting $z-\zeta$ which means that $\operatorname{det}(\mathbf{T})^{-1}$ lies in $o_{L} \llbracket z \rrbracket\left[\frac{1}{z-\zeta}\right]$. Say we have a relation $(z-\zeta)^{e}=\operatorname{det}(\mathbf{T}) u$ in $o_{L} \llbracket z \rrbracket$, for some $e \geq 0$ and some $u \in o_{L} \llbracket z \rrbracket$. By a comparison of powers of $z-\zeta$, we 
may assume that $u$ is not divisible by $z-\zeta$. In this equation there is only one prime element of $o_{L} \llbracket z \rrbracket$ occurring on both sides, which, by factoriality, implies that $u$ has to be a unit in $o_{L} \llbracket z \rrbracket$. Let $(z-\zeta)^{e^{\prime}}=\operatorname{det}\left(\mathbf{T}^{\prime}\right) u^{\prime}$ be the corresponding relation for the local shtuka $\hat{M}$, with a unit $u^{\prime} \in o_{L} \llbracket z \rrbracket^{\times}$and some suitable $e^{\prime} \geq 0$. Since $\hat{\mathcal{M}} \rightarrow \hat{M}$ becomes an isomorphism after inverting $\pi$, we see that $\operatorname{det}(\mathbf{A}) \in$ $o_{L} \llbracket z \rrbracket[1 / \pi]^{\times}$. Note that the natural reduction-mod- $z$ map $o_{L} \llbracket z \rrbracket \rightarrow o_{L}, h \mapsto h(0)$, induces an epimorphism of Abelian groups $o_{L} \llbracket z \rrbracket\left[\frac{1}{\pi}\right]^{\times} \rightarrow L^{\times}$, so that the absolute term $\delta:=\operatorname{det}(\mathbf{A})(0)$ of $\operatorname{det}(\mathbf{A})$ lies in $L^{\times}$. By virtue of the relations derived above, the equation $\operatorname{det}(\mathbf{A}) \operatorname{det}(\mathbf{T})=\operatorname{det}\left(\mathbf{T}^{\prime}\right) \sigma(\operatorname{det}(\mathbf{A}))$ yields

$$
\operatorname{det}(\mathbf{A}) u^{-1}(z-\zeta)^{e}=u^{\prime-1}(z-\zeta)^{e^{\prime}} \sigma(\operatorname{det}(\mathbf{A}))
$$

which modulo $z$ gives $\delta^{q-1}=\frac{u^{\prime}(0)}{u(0)}(-\zeta)^{e-e^{\prime}}$ in $L^{\times}$. Suppose for a moment that $e=e^{\prime}$. In this case it follows at once that $\delta$ is a unit in $o_{L}$, so that $\operatorname{det}(\mathbf{A})$ is a unit in $o_{L} \llbracket z \rrbracket$. Therefore it remains to verify that our assumption $e=e^{\prime}$ is justified. This can be seen as follows: The reduction-mod- $\pi$ map $o_{L} \llbracket z \rrbracket \rightarrow \ell \llbracket z \rrbracket$ is an epimorphism with kernel $\pi o_{L} \llbracket z \rrbracket$, and via applying the functor $\cdot \otimes_{o_{L} \llbracket z \rrbracket} \ell \llbracket z \rrbracket$ to $F_{\hat{M}}: \sigma^{*} \hat{M} \rightarrow \hat{M}$ we obtain a commutative diagram

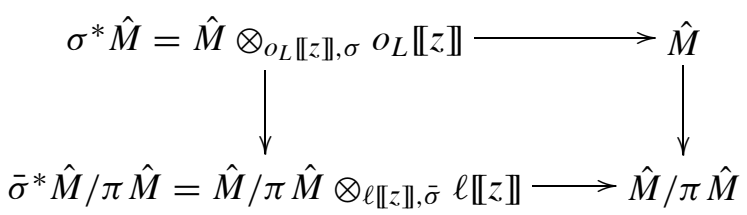

where in the upper row (respectively, the bottom row) both modules are finite free of the same rank over $o_{L} \llbracket z \rrbracket$ (respectively, over $\left.\ell \llbracket z \rrbracket\right)$ and the arrow is given by $F_{\hat{M}}$ (respectively, by $\left.\bar{F}=F_{\hat{M}} \otimes \mathrm{id}_{\ell \llbracket z \rrbracket}\right)$. The reduced matrix $\overline{\mathbf{T}^{\prime}} \in \ell \llbracket z \rrbracket^{s \times s}$ describes the map $\bar{F}$ with respect to the $\ell \llbracket z \rrbracket$-bases $\overline{\sigma^{*} \mathfrak{C}}=\bar{\sigma}^{*} \overline{C^{*}}$ of $\bar{\sigma}^{*} \hat{M} / \pi \hat{M}$ and $\overline{\mathfrak{C}}$ of $\hat{M} / \pi \hat{M}$ respectively, and from what we have seen before, we derive the relation $\operatorname{det}\left(\overline{\mathbf{T}^{\prime}}\right) \overline{u^{\prime}}=z^{e^{\prime}}$, i.e. $e^{\prime}=\operatorname{ord}_{z}\left(\operatorname{det}\left(\overline{\mathbf{T}^{\prime}}\right)\right)$, the latter being true since $\overline{u^{\prime}} \in \ell \llbracket z \rrbracket^{\times}$. In particular we have $\operatorname{det}\left(\overline{\mathbf{T}^{\prime}}\right) \in \ell \llbracket z \rrbracket-\{0\}$. A similar observation for the local shtuka $\hat{\mathcal{M}}$ instead of $\hat{M}$ shows that $e=\operatorname{ord}_{z}(\operatorname{det}(\overline{\mathbf{T}}))$. Let $C=\operatorname{coker}\left(F_{\hat{\mathcal{M}}}\right)$ and $C^{\prime}=\operatorname{coker}\left(F_{\hat{M}}\right)$. Multiplication with the matrix $\overline{\mathbf{T}^{\prime}}$ gives rise to a finite presentation $\ell \llbracket z \rrbracket^{s} \rightarrow \ell \llbracket z \rrbracket^{s} \rightarrow C^{\prime} / \pi C^{\prime} \rightarrow 0$. Taking determinants in an equation of the form $\mathbf{S}_{1} \overline{\mathbf{T}^{\prime}} \mathbf{S}_{2}=\operatorname{Diag}\left(a_{1}, \ldots, a_{d}, 0,0, \ldots, 0\right)$, where $\mathbf{S}_{1}, \mathbf{S}_{2} \in \mathrm{Gl}_{s}(\ell \llbracket z \rrbracket)$ are suitable matrices such that $a_{1}, \ldots, a_{d} \in \ell \llbracket z \rrbracket-\{0\}$ are the elementary divisors of $\overline{\mathbf{T}^{\prime}}$ (see [7], VII.4.5.1), yields that necessarily $d=s$, so that $C^{\prime} / \pi C^{\prime}$ is a torsion $\ell \llbracket z \rrbracket$-module and

$$
C^{\prime} / \pi C^{\prime} \cong \ell \llbracket z \rrbracket / a_{1} \ell \llbracket z \rrbracket \oplus \ldots \oplus \ell \llbracket z \rrbracket / a_{s} \ell \llbracket z \rrbracket \cong \ell^{n_{1}} \oplus \ldots \oplus \ell^{n_{s}}
$$

where $n_{j}=\operatorname{ord}_{z}\left(a_{j}\right)$ and $\sum_{j} n_{j}=e^{\prime}$, i.e. $e^{\prime}=\operatorname{ord}_{z}\left(\operatorname{det}\left(\overline{\mathbf{T}^{\prime}}\right)\right)=\operatorname{rk}_{\ell}\left(C^{\prime} / \pi C^{\prime}\right)=$ $\mathrm{rk}_{O_{L}}\left(C^{\prime}\right)$, the latter equation being valid since $C^{\prime} / \pi C^{\prime} \cong C^{\prime} \otimes_{o_{L} \llbracket z \rrbracket} \ell \llbracket z \rrbracket$. Finally, 
imitating this argument for the local shtuka $\hat{\mathcal{M}}$ yields that $e=\operatorname{ord}_{z}(\operatorname{det}(\overline{\mathbf{T}}))=$ $\mathrm{rk}_{\ell}(C / \pi C)=\mathrm{rk}_{O_{L}}(C)$. So it remains to show that $\mathrm{rk}_{O_{L}}(C)=\mathrm{rk}_{O_{L}}\left(C^{\prime}\right)$. Indeed, we know that $\psi: \hat{\mathcal{M}} \rightarrow \hat{M}$ gives back $f$ in the generic fiber, which means that $\psi$ is an isomorphism after inverting $\pi$. Therefore, inverting $\pi$ in the commutative diagram with exact rows

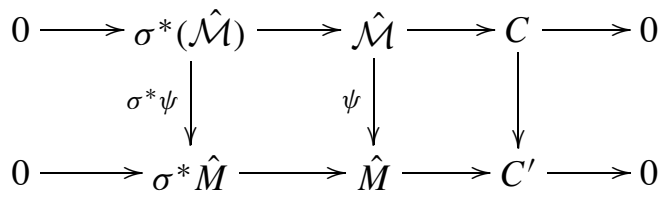

exhibits $\left(\sigma^{*} \psi\right)[1 / \pi]=\sigma^{*}(\psi[1 / \pi])$ and $\psi[1 / \pi]$ as $o_{L} \llbracket[z \rrbracket[1 / \pi]$-linear isomorphisms, so that the Snake Lemma yields $C^{\prime}[1 / \pi] \cong C[1 / \pi]$, and we obtain $\mathrm{rk}_{o_{L}}\left(C^{\prime}\right)=\operatorname{dim}_{L}\left(C^{\prime}[1 / \pi]\right)=\operatorname{dim}_{L}(C[1 / \pi])=\mathrm{rk}_{O_{L}}(C)$, as desired.

\section{The reduction criterion for Anderson motives}

Definition 6.1. (a) Let $\underline{\mathcal{M}} \in \operatorname{FMod}\left(A_{o_{L}}\right)$. Following Gardeyn [13], $\underline{\mathcal{M}}$ is called $A_{o_{L}}$-maximal if for every $\underline{\mathcal{N}} \in \operatorname{FMod}\left(A_{o_{L}}\right)$ the canonical map

$$
\left.\operatorname{Hom} F \operatorname{Mod}\left(A_{o_{L}}\right)(\underline{\mathcal{N}}, \underline{\mathcal{M}}) \rightarrow \operatorname{Hom} F \operatorname{Mod}\left(A_{L}\right) \underline{(\mathcal{N}}[1 / \pi], \underline{\mathcal{M}}[1 / \pi]\right)
$$

is surjective (and hence bijective).

(b) An object $\underline{\mathcal{M}^{\prime}} \in \operatorname{FMod}\left(A_{o_{L}, \pi}\right)$ is called $A_{o_{L}, \pi}$-maximal if for every $\underline{\mathcal{N}}^{\prime} \in$ $\operatorname{FMod}\left(A_{O_{L}, \pi}\right)$ the canonical map

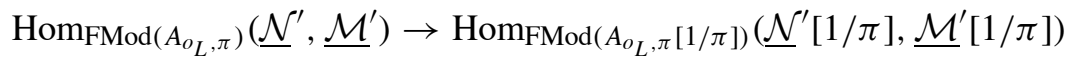

is surjective (and hence bijective).

(c) Let $\underline{M} \in \operatorname{FMod}\left(A_{L}\right)$. An object $\underline{\mathcal{M}} \in \operatorname{FMod}\left(A_{o_{L}}\right)$ is called an $A_{o_{L}}$-maximal model for $\underline{M}$ if $\underline{\mathcal{M}}[1 / \pi] \cong \underline{M}$ inside $\operatorname{FMod}\left(A_{L}\right)$ (i.e. $\underline{\mathcal{M}}$ is a model for $\underline{M}$ ) and if $\underline{M}$ is $A_{o_{L}}$-maximal. Correspondingly, given $\underline{M}^{\prime} \in \operatorname{FMod}\left(A_{o_{L}, \pi}[1 / \pi]\right)$, an object $\underline{\mathcal{M}^{\prime}} \in \operatorname{FMod}\left(A_{o_{L}, \pi}\right)$ is called an $A_{o_{L}, \pi}$-maximal model for $\underline{M}^{\prime}$ if $\underline{\mathcal{M}}[1 / \pi] \cong \underline{M}^{\prime}$ inside $\operatorname{FMod}\left(A_{o_{L}, \pi}[1 / \pi]\right)$ and if $\underline{\mathcal{M}}^{\prime}$ is $A_{o_{L}, \pi}$-maximal. [13].

The existence of ( $A_{O_{L}}$ and $A_{o_{L}, \pi^{-}}$)maximal models has been established in

Proposition 6.2 ([13, Proposition 2.13]). Let $\underline{M} \in \operatorname{FMod}\left(A_{L}\right)$. Then the following assertions hold:

(i) $\underline{M}$ admits an $A_{o_{L}}$-maximal model, which is unique up to unique isomorphism;

(ii) If a model $\underline{\mathcal{M}} \in \operatorname{FMod}\left(A_{o_{L}}\right)$ of $\underline{M}$ is good in the weak sense of Definition 4.5, then it is $A_{o_{L}}$-maximal.

The next proposition is a variant of Gardeyn's theory of maximal models. 
Proposition 6.3. The following assertions hold:

(i) Every $\underline{M} \in \operatorname{FMod}\left(A_{o_{L}, \pi}[1 / \pi]\right)$ admits a maximal model, which is unique up to unique isomorphism;

(ii) If $\underline{M} \in \operatorname{FMod}\left(A_{L}\right)$ is given and if $\underline{\mathcal{M}} \in \operatorname{FMod}\left(A_{o_{L}}\right)$ is an $A_{o_{L}}$-maximal model of $\underline{M}$ then $\underline{\mathcal{M}} \otimes_{A_{o_{L}}} A_{o_{L}, \pi} \in \operatorname{FMod}\left(A_{o_{L}, \pi}\right)$ is an $A_{o_{L}, \pi}$-maximal model of $\underline{M} \otimes_{A_{L}} A_{o_{L}, \pi}[1 / \pi] \in \operatorname{FMod}\left(A_{o_{L}, \pi}[1 / \pi]\right)$;

(iii) Let $\underline{M} \in \operatorname{FMod}\left(A_{o_{L}, \pi}[1 / \pi]\right)$ and let $\underline{\mathcal{M}} \in \operatorname{FMod}\left(A_{o_{L}, \pi}\right)$ be a model of $\underline{M}$. If $\underline{\mathcal{M}}$ is a good model in the weak sense of Definition 4.5 , then it is $A_{o_{L}, \pi^{-}}$ maximal.

Proof. For (i) (respectively (ii); respectively (iii)), see [13], 3.3(i) (respectively 3.4(i); respectively $2.13(\mathrm{ii})$ ). Note that strictly speaking Gardeyn proves these statements for the rings $\Gamma\left(\mathfrak{A}(\infty), \mathcal{O}_{\mathfrak{A}(\infty)}\right)$ instead of $A_{o_{L}, \pi}[1 / \pi]$ and $\Gamma\left(\mathfrak{A}(\infty), \mathcal{O}_{\mathfrak{A}(\infty)}\right) \cap$ $A_{o_{L}, \pi}$ instead of $A_{o_{L}, \pi}$. His arguments carry over literally to our rings.

We may conclude:

Proposition 6.4. In the weak sense of Definition 4.5 a Frobenius $A_{L}$-module $\underline{M}$ admits a good model over $A_{o_{L}}$ if and only if $\underline{M} \otimes_{A_{L}} A_{o_{L}, \pi}[1 / \pi] \in \operatorname{FMod}\left(A_{o_{L}, \pi}[1 / \pi]\right)$ admits a good model over $A_{o_{L}, \pi}$. If this is the case, the functor $(\underline{\mathcal{M}}, \alpha) \mapsto$ $\left(\underline{\mathcal{M}} \otimes_{A_{o_{L}}} A_{o_{L}, \pi}, \alpha \otimes \mathrm{id}_{A_{o_{L}, \pi}[1 / \pi]}\right)$ is an equivalence of categories between the good models of $\underline{M}$ and the good models of $\underline{M} \otimes_{A_{L}} A_{o_{L}, \pi}[1 / \pi]$.

Proof. First suppose that $\underline{M}$ admits a good model $\underline{\mathcal{M}} \in \operatorname{FMod}\left(A_{o_{L}}\right)$. It follows that $\underline{\mathcal{M}}$ is an $A_{o_{L}}$-maximal model of $\underline{M}$. Furthermore, its image $\underline{\mathcal{M}} \otimes_{A_{o_{L}}} A_{o_{L}, \pi}$ inside $\operatorname{FMod}\left(A_{o_{L}, \pi}\right)$ is an $A_{o_{L}, \pi}$-maximal model of $\underline{M} \otimes_{A_{L}} A_{o_{L}, \pi}[1 / \pi]$. Since the reduction of $\mathcal{M}$ is canonically isomorphic to the reduction of $\underline{\mathcal{M}} \otimes_{A_{o_{L}}} A_{o_{L}, \pi}$ by Proposition 4.9, it follows that the latter is a good model.

Conversely, suppose that $\underline{M} \otimes_{A_{L}} A_{o_{L}, \pi}[1 / \pi]$ admits a good model $\underline{\widehat{\mathcal{M}}} \in$ $\operatorname{FMod}\left(A_{o_{L}, \pi}\right)$. Necessarily $\widehat{\widehat{\mathcal{M}}}$ is a maximal model by Proposition 6.3(iii). We know that there is an $A_{O_{L}}$-maximal model $\underline{\mathcal{M}} \in \operatorname{FMod}\left(A_{O_{L}}\right)$ of $\underline{M}$ such that $\underline{\mathcal{M}} \otimes_{A_{o_{L}}} A_{o_{L}, \pi} \cong \underline{\widehat{\mathcal{M}}}$, and that the reduction of $\underline{\mathcal{M}}$ is canonically isomorphic to the reduction of $\underline{\mathcal{M}}$ by Propositions 6.2,6.3(ii) and 4.9. Since $\underline{\widehat{\mathcal{M}}}$ is a good model, so is $\mathcal{M}$. This proves the first statement and it also proves essential surjectivity of the functor.

To prove full faithfulness let $(\underline{\mathcal{M}}, \alpha)$ and $\left(\underline{\mathcal{M}}, \alpha^{\prime}\right)$ be good models of $\underline{M}$ and let $\hat{\beta}: \underline{\mathcal{M}} \otimes_{A_{o_{L}}} A_{o_{L}, \pi} \stackrel{\sim}{\longrightarrow} \underline{\mathcal{M}^{\prime}} \otimes_{A_{o_{L}}} A_{o_{L}, \pi}$ be an isomorphism in $\operatorname{FMod}\left(A_{o_{L}, \pi}\right)$ satisfying $\alpha^{\prime} \otimes \mathrm{id}=\hat{\beta} \circ(\alpha \otimes \mathrm{id})$. Since $A_{o_{L}}=A_{L} \cap A_{o_{L}, \pi}$ inside $A_{o_{L}, \pi}[1 / \pi]$, we can recover $\mathcal{M}$ as $\mathcal{M}=\alpha(M) \cap \mathcal{M} \otimes_{A_{o_{L}}} A_{o_{L}, \pi}$. This implies $\hat{\beta}(\mathcal{M})=\mathcal{M}^{\prime}$ and $\beta:=\left.\hat{\beta}\right|_{\mathcal{M}}$ is the desired isomorphism $\beta: \underline{\mathcal{M}} \stackrel{\sim}{\longrightarrow} \underline{\mathcal{M}}$ with $\alpha^{\prime}=\beta \circ \alpha$. This proves full faithfulness. 
For Anderson $A$-motives Proposition 6.4 and Theorem 4.7 imply the following:

Corollary 6.5. Let $\underline{M}$ be an Anderson A-motive over L. Then in the strong sense of Definition 4.6, $\underline{M}$ admits a good model $\underline{\mathcal{M}}$ if and only if the associated analytic Anderson $A(1)$-motive $\underline{M} \otimes_{A_{L}} A_{o_{L}, \pi}[1 / \pi]$ admits a good model $\underline{\mathcal{M}}^{\prime}$. If this is the case, the functor $(\underline{\mathcal{M}}, \alpha) \mapsto\left(\underline{\mathcal{M}} \otimes_{A_{o_{L}}} A_{o_{L}, \pi}, \alpha \otimes \mathrm{id}_{A_{o_{L}, \pi}[1 / \pi]}\right)$ is an equivalence of categories between the good models of $\underline{M}$ and the good models of $\underline{M} \otimes_{A_{L}} A_{o_{L}, \pi}[1 / \pi]$.

This corollary together with Theorem 5.3 and Corollary 5.5 implies the following criterion for good reduction of Anderson $A$-motives, which can be regarded as an analog of the reduction criteria for Abelian varieties of Grothendieck [15, Proposition IX.5.13] and de Jong [19, 2.5].

Corollary 6.6. Let $\underline{M}$ be an Anderson A-motive over $L$ such that $\operatorname{coker}\left(F_{M}\right)$ is annihilated by $\mathfrak{J}^{d}$ for some $d$. Then the following assertions are equivalent:

(i) $\underline{M}$ admits a good model $(\underline{\mathcal{M}}, \alpha)$ in the strong sense of Definition 4.6 , i.e. there is an object $\underline{\mathcal{M}} \in \operatorname{FMod}\left(A_{o_{L}}\right)$ such that $\operatorname{coker}\left(F_{\mathcal{M}}\right)$ is a finite free $o_{L}$-module and is annihilated by $\mathfrak{J}^{d}$, together with an isomorphism $\alpha: \underline{M} \stackrel{\sim}{\longrightarrow}[1 / \pi]$ inside FMod $\left(A_{L}\right)$;

(ii) There is an effective local shtuka $\underline{\hat{M}}$ at $\varepsilon$ over $o_{L}$ such that $\operatorname{coker}\left(F_{\hat{M}}\right)$ is annihilated by $\mathfrak{J}^{d}$, and an isomorphism $\underline{M} \otimes_{A_{L}} A_{o_{L},(\varepsilon, \pi)}[1 / \pi] \cong \underline{\hat{M}}[1 / \pi]$ inside $\operatorname{FMod}\left(A_{o_{L},(\varepsilon, \pi)}[1 / \pi]\right)$.

Moreover, there is an equivalence of categories

$$
\begin{aligned}
& \left\{\begin{array}{l}
\text { good models }(\mathcal{M}, \alpha) \text { of } \underline{M} \text { in the } \\
\text { sense of Definitions } 4.6 \text { and } 4.5
\end{array}\right\} \\
& \stackrel{\sim}{\longleftrightarrow}\left\{\begin{array}{l}
\text { pairs }(\underline{\hat{M}}, f) \text { consisting of } \\
\bullet \text { a local shtuka } \hat{\hat{M}} \text { at } \varepsilon \text { over } o_{L}, \text { and } \\
\bullet \text { an isomorphism in } \operatorname{FMod}\left(A_{o_{L},(\varepsilon, \pi)}[1 / \pi]\right) \\
f: \underline{M} \otimes_{A_{L}} A_{o_{L},(\varepsilon, \pi)}[1 / \pi] \stackrel{\sim}{\longrightarrow}[1 / \pi]
\end{array}\right\} \\
& (\underline{\mathcal{M}}, \alpha) \longmapsto(\underline{\mathcal{M}}, \alpha) \otimes_{A_{o_{L}}} A_{o_{L},(\varepsilon, \pi)},
\end{aligned}
$$

where on the right-hand side a morphism of pairs $\hat{\beta}:(\underline{\hat{M}}, f) \stackrel{\sim}{\longrightarrow}\left(\underline{\hat{M}}^{\prime}, f^{\prime}\right)$ is defined to be an isomorphism of local shtukas $\hat{\beta}: \underline{\hat{M}} \stackrel{\sim}{\longrightarrow} \underline{\hat{M}^{\prime}}$ satisfying $f^{\prime}=$ $\hat{\beta} \circ f$.

\section{References}

[1] G. ANDERSON $t$-motives, Duke Math. J. 53 (1986), 457-502.

[2] G. BöCKLE and U. HARTL, Uniformizable families of t-motives, Trans. Amer. Math. Soc. 359 (2007), 3933-3972. 
[3] M. Bornhofen and U. Hartl Pure Anderson motives and Abelian $\tau$-sheaves, Math. Z. 268 (2011), 67-100.

[4] S. Bosch, "Lectures on Formal and Rigid Geometry", Lecture Notes in Math., Vol. 2105, Springer-Verlag, Berlin, 2014.

[5] S. Bosch, U. GÜNTZER and R. REMmert, "Non-Archimedean Analysis", Grundlehren, Vol. 261, Springer-Verlag, Berlin, 1984.

[6] S. BosCH and W. LÜTKeBOHMERT, Formal and rigid geometry I. Rigid spaces, Math. Ann. 295 (1993), 291-317.

[7] N. BourbaKi, "Eléments de mathématique - Algèbre", Masson, Paris, 1981.

[8] N. Bourbaki, "Eléments de mathématique - Algèbre Commutative", Hermann, Paris, 1967.

[9] V. G. DRINFELD, Elliptic modules, Math. USSR-Sb. 23 (1976), 561-592.

[10] D. EISEnBud, "Commutative Algebra with a View Toward Algebraic Geometry", GTM Vol. 150, Springer-Verlag, Berlin, 1995.

[11] J. FRESNEL and M. VAN DER PUT, "Géométrie analytique rigide et applications", Progress in Mathematics, Vol. 218, Birkhäuser, Basel, 2004.

[12] F. GARDEYN, A Galois criterion for good reduction of $\tau$-sheaves, J. Number Theory 97 (2002), 447-471.

[13] F. GARDEYN, The structure of analytic $\tau$-sheaves, J. Number Theory 100 (2003), 332-362.

[14] A. Grothendieck, "Élements de géométrie algébrique", Publ. Math. IHES, Vol. 4, 8, 11 , 17, 20, 24, 28, 32, Bures-Sur-Yvette, 1960-1967; see also Grundlehren, Vol. 166, SpringerVerlag, Berlin, 1971.

[15] P. Deligne, A. Grothendieck et al., "SGA 7: Groupes de monodromie en géométrie algébrique", LNM, Vol. 288, Springer, Berlin-Heidelberg, 1972.

[16] U. HARTL, A dictionary between Fontaine-theory and its analogue in equal characteristic, J. Number Theory 129 (2009), 1734-1757.

[17] U. HARTL, Period spaces for Hodge structures in equal characteristic, Ann. of Math. 173 (2011), 1241-1358.

[18] U. HARTL and R. K. SINGH, Local shtukas and divisible local Anderson-modules, in preparation.

[19] A. J. DE JoNG, Homomorphisms of Barsotti-Tate groups and crystals in positive characteristic, Invent. Math. 134 (1998), 301-333.

[20] H. Matsumura, "Commutative Ring Theory", Cambridge Studies in Advanced Mathematics, Vol. 8, Cambridge University Press, 1986.

[21] H. MATZAT, Introduction to Drinfeld modules, In: "Drinfeld Modules, Modular Schemes and Applications" (Alden-Biesen, 1996), World Sci. Publishing, River Edge, NJ, 1997, $3-$ 16.

[22] J.-P. SerRe and J. TATE, Good reduction of Abelian varieties, Ann. of Math. 88 (1968), 492-517.

Universität Münster

Mathematisches Institut

Einsteinstr. 62

D - 48149 Münster, Germany

urs.hartl@uni-muenster.de 